\title{
A Coherent Approach for Interrogating Polybenzoxazole Fibers for Residual Phosphoric Acid
}
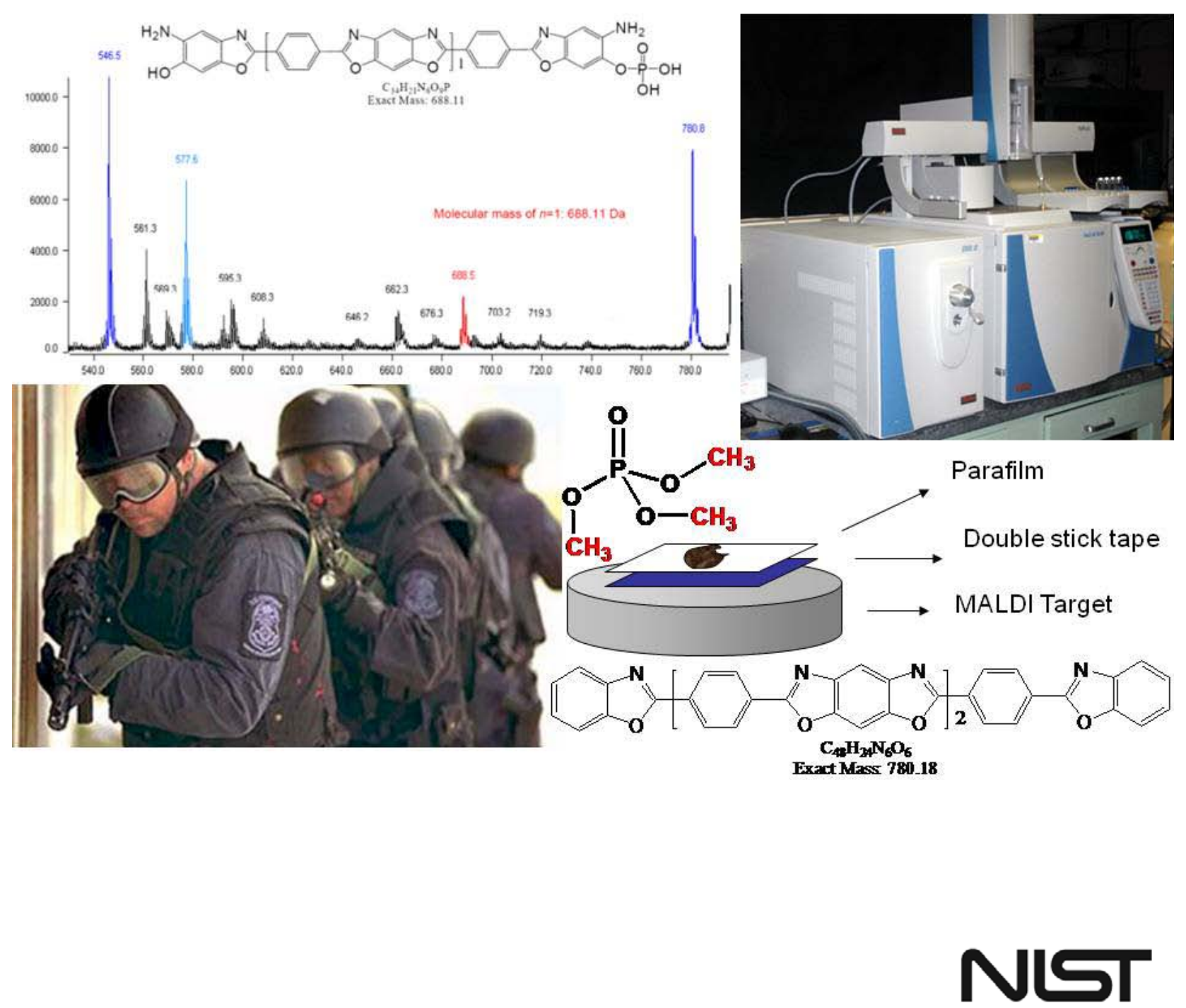

National Institute of Standards and Technology U.S. Department of Commerce 
NISTIR 7584

\title{
A Coherent Approach for Interrogating Polybenzoxazole Fibers for Residual Phosphoric Acid
}

\author{
Gale Holmes, Eun Su Park, \\ Charles Guttman and Kathleen Flynn
}

Characterization and Measurement Group Polymers Division

Materials Science and Engineering Laboratory

John Sieber

Inorganic Chemical Metrology Group Analytical Chemistry Division

Chemical Science and Technology Laboratory

Stephanie Watson Spectrochemical Methods Group Materials and Construction Research Division Building and Fire Research Laboratory

Kirk Rice

Office of Law Enforcement Standards Electronics and Electrical Engineering Laboratory

December 2009



U.S. Department of Commerce

Gary Locke, Secretary

National Institute of Standards and Technology Patrick D. Gallagher, Deputy Director 


\begin{abstract}
Because of the premature failure of in-service soft-body armor that contains the ballistic fiber poly[(benzo-[1,2-d:5,4-d’]-benzoxazole-2,6-diyl)-1,4-phenylene] (PBO), the Office of Law Enforcement Standards (OLES) at the National Institute of Standards and Technology (NIST) initiated a research program to investigate the reasons for the failure and to develop testing methodologies and protocols to ensure that these types of failures do not reoccur. In a report that focused on the stability of the benzoxazole ring that is characteristic of PBO fibers, Holmes et al. (2006) showed that the benzoxazole ring was susceptible to hydrolytic degradation under acid conditions. Because of the processing conditions for the fibers, it is suspected by many researchers that residual phosphoric acid may cause the degradation of the benzoxazole ring that result in a reduction in ballistic performance. Prior to this work, no definitive data have indicated the presence of phosphoric acid since the residual phosphorus is not easily extracted and the processed fibers are known to be laced with phosphorus containing processing aids. Methods to efficiently extract phosphorus from PBO are described in this report. Further, characterization determined that the majority of the extractable phosphorus in PBO was attributed to the octyldecyl phosphate processing aid with some phosphoric acid being detected. Analysis by matrix assisted laser desorption ionization of model PBO oligomers indicates that the non-extractable phosphorus is attached to the PBO polymer chain as a monoaryl phosphate ester. The response of model aryl phosphates to $\mathrm{NaOH}$ exposure indicates that the monoaryl phosphate ester is stable to the $\mathrm{NaOH}$ washes used in the manufacturing process to neutralize the phosphoric acid reaction medium and extract residual phosphorus impurities. These results strongly indicate that phosphoric acid that is chemically bound to the PBO molecule as a monoaryl phosphate ester is the primary cause for the hydrolytic degradation and that this residual acid type is not readily removed by caustic washes.
\end{abstract}

\title{
Acknowledgements
}

The authors wish to thank the National Institute of Standards and Technology Office of Law Enforcement and Standards (NIST-OLES) under the auspices of the National Institute of Justice (NIJ) for funding this work under interagency agreement 2003-IJ-R-029. NIJ is not responsible for the content of this article. 


\section{INTRODUCTION}

The poly[(benzo-[1,2-d:5,4-d']-benzoxazole-2,6-diyl)-1,4-phenylene] (PBO) fiber is part of a sub-class of rigid-rod polymers known as polybenzazoles (PBXs). PBO evolved out of the pioneering research of Vogel and Marvel [1,2] on thermally stable polybenzimidazole (PBI), a related PBX polymer. Although PBO can be found in a variety of applications, the primary use for this polymer has been in the manufacture of soft body armor (SBA) for civilian first responder applications, where its superior properties relative to polyaramids (i.e., Kevlar, Twaron) [3] ushered in the development of ultra-lightweight SBA.

PBO is prepared by the reaction of 1,3-diamino-4,6-dihydroxybenzene (DADHB) dihydrochloride with terephthalic acid (TA) in a reaction medium consisting of polyphosphoric acid (PPA) enriched with $\mathrm{P}_{2} \mathrm{O}_{5}$ as a dehydrating agent. Although the fibers have superior tensile strength and modulus, cut and abrasion resistance, and flame retardation relative to other high performance fibers, concerns have recently been expressed recently about the long-term stability of PBO fibers [4]. Several studies indicate a reduction in the mechanical properties of PBO (i.e., strain-to-failure, ultimate tensile strength) when exposed to moisture [3,5]. To better understand these concerns, the reaction scheme adapted from the research of So et al. [6,7] is shown in Figure 1, where the key feature in the PBO reaction is the formation of the benzoxazole ring structure. The influences of $\mathrm{pH}$, ultraviolet (UV) radiation, and other factors on the stability of this ring have recently been reviewed by Holmes et al. [8], with literature results indicating that the acid-catalyzed pathway to hydrolysis of the benzoxazole ring in $\mathrm{PBO}$ is feasible, but $\mathrm{pH}$ sensitive.

In the reaction scheme shown in Figure 1, the carbonyl group in TA initially reacts with PPA to form a carboxylic-phosphoric mixed anhydride (Species A). The hydroxyl groups of protonated DADHB, which are also in equilibrium with PPA as DADHB-PPA (aryl-phosphate) esters, reacts with the mixed anhydride to form esters (Species B). Species B then undergoes the expected Railford acyl migration [8] to form an amide prior to the ring closure reaction that forms the benzoxazole ring (Species C). Reaction of Species C, 2,2'-(1,4-phenylene)bis(5amino-6-benzoxazolol), with Species A leads to the formation of the PBO polymer where the PBO oligomers are preferentially capped with DADHB. The terminal DADHB groups have un- 
reacted hydroxyl groups in the reaction medium that are in dynamic equilibrium (i.e., partially reacted) with PPA to form aryl-phosphate esters.

Elemental analysis indicates that processed PBO fibers have a residual phosphorus (P) mass fraction of approximately $0.3 \%$ to $0.4 \%[3,4,9,10]$. This observation and the synthesis of PBO in PPA have led many researchers to make the plausible assumption that the residual phosphorus in PBO fibers is due to phosphoric acid (PA) and to infer that PA catalyzes the mechanical property degradation observed in PBO fibers. This assumption is based on the research of Jackson et al. [11] who showed that under acidic conditions simple benzoxazoles hydrolyze principally to the corresponding amidophenols. Two research reports [4,5] provide indirect evidence of the PA catalyzed hydrolysis reaction. In refererence 5, the tensile strengths of PBO fibers are found to decrease when phosphorus mass fraction is increased above $1 \%$ by exposing the fibers to increasing amounts of phosphoric acid. In the manufacture of PBO fibers, a residual phosphorus level between (2000 to $5000 \mathrm{mg} / \mathrm{kg}$ ) is generally targeted [12]. This is particularly important when one observes that the severe conditions achieved by exposing PBO fibers to phosphoric acid are unlikely to be encountered in normal applications.

Furthermore, it is noted that extreme washing treatments (boiling water or Soxhlet extraction) are not effective in lowering the phosphorous content in as-spun dried or heat treated fiber samples. Research by others [9] indicates that the residual phosphorus mass fraction in PBO fibers can be reduced from $0.4 \%$ mass fraction to just $0.25 \%$ mass fraction by washing the fibers with supercritical carbon dioxide. Finally, a recent investigation performed at the National Institute of Standards and Technology (NIST) exposed soft body armors composed of PBO fibers to a constant hydrolytic environment for six months. Consistent with previous research, chemical degradation of the fibers was observed by difference Fourier transform infrared (FTIR) spectroscopy [10].

All extant research [3-5,10,13] indicates that PBO fibers are degraded by hydrolytic action, which researchers presume to be catalyzed by residual PA. Complicating this seemingly straight forward interpretation is the absence of direct evidence for the existence of PA in processed PBO fibers and the knowledge that alkyl-phosphate esters are often used as processing aids during the manufacture of PBO fibers. Therefore, the research in this report will focus on the development of a direct approach for identifying phosphoric acid in the presence of alkyl- 
phosphate esters that may be present and to provide additional insight as to the nature of the seemingly non-extractable phosphorus found in PBO fibers.

In this report, X-ray fluorescence (XRF) spectrometry along with standard derivatization procedures, extraction techniques, and model compounds are used to develop a coherent approach for interrogating PBO fibers for the presence of residual phosphoric acid compounds. Because of the insolubility of PBO oligomers and pre-polymeric species in most solvents, matrix assisted laser desorption/ionization time-of-flight mass spectrometry (MALDI-TOF MS) is also employed to identify species in polymer oligomers. A dual injector gas chromatograph/mass spectrometer (GC/MS) is used in electron impact (EI) and chemical ionization (CI) modes to obtain fragmentation spectra and molecular ion data for extracted low molecular mass species. To unequivocally identify phosphorus extracted compounds, a parallel flame photometric detector (FPD) and a non-parallel nitrogen phosphorus detector (NPD) are also used.

\section{EXPERIMENTAL}

Materials: 2,2'-(1,4-phenylene) bis(benzoxazolyl) (AF1), 2,6-diphenylbenzo[1,2-d;5,4d']bisoxazole (AF2), and 2-phenylbenzoxazole (AF3), model compounds of PBO, were supplied by the Wright Patterson Air Force Research Laboratories (see Figure 2), with the relationship of these model compounds to the PBO chain structure shown in Figure 3. PBO fibers were obtained from a field returned vest supplied by the Department of Justice. Phosphoric acid, phosphorous pentoxide, 2-phenylbenzoxazole (PBOm), 4,6-diaminoresorcinol (DADHB), 2aminophenol (2-AP), terephthalic acid (TA), trimethyl phosphate (TMP), triphenyl phosphate (TPhP), sodium phenyl phosphate dibasic dihydrate (DNaPhP), sodium hydroxide and concentrated hydrochloric acid ( $\mathrm{HCl}, 37$ \% mass fraction) were purchased from Aldrich Chemical Co. ${ }^{1}$ and used as received. HCl used in this study has phosphorus $(\mathrm{P})<0.01 \mathrm{mg} / \mathrm{kg}$ (Fluka data).

\footnotetext{
${ }^{1}$ Certain commercial products and equipment were named in this paper for the purpose of adequately specifying the experimental conditions and the sources of analytical results. Such descriptions do not constitute endorsement by the National Institute of Standards and Technology, nor do they imply that the equipment and products are necessarily the best for the purpose.
} 
Synthesis of PBO oligomer: The synthesis method was adapted from references 14 and 15 . In this study, 2-AP was added as an end-capping material to keep the molecular mass of the PBO oligomer in a range that could be analyzed by MALDI-TOF MS. Poly(phosphoric acid) was prepared by the following method. Phosphorous pentoxide (500 g) was added slowly to $196 \mathrm{~mL}$ of $85 \%$ PA while the mixture was stirred under nitrogen. The mixture was then heated at $150{ }^{\circ} \mathrm{C}$ for $12 \mathrm{~h}$ to yield a homogeneous (clear) solution of PPA.

After the temperature of PPA was decreased to $80{ }^{\circ} \mathrm{C}, 5.00 \mathrm{~g}(0.0235 \mathrm{~mol})$ of 4,6-diaminoresorcinol and $0.51 \mathrm{~g}(0.0047 \mathrm{~mol})$ of 2-AP were added under a slow stream of nitrogen, and the mixture was heated at $80^{\circ} \mathrm{C}$ for $16 \mathrm{~h}$. Then the mixture was heated at $110{ }^{\circ} \mathrm{C}$ for $8 \mathrm{~h}$ until the evolution of $\mathrm{HCl}$ ceased. Terephthalic acid (TA) in the amount of $4.29 \mathrm{~g}$ ( $0.0258 \mathrm{~mol})$ was added, and the mixture was heated at $150{ }^{\circ} \mathrm{C}$ for $12 \mathrm{~h}$ followed by $175^{\circ} \mathrm{C}$ for $4 \mathrm{~h}$. The brown solution was poured into water and washed with distilled water and dried. The oligomer was then washed 10 times with $1 \mathrm{~L}$ aliquots of $52{ }^{\circ} \mathrm{C}$ water, dried, and rewashed 10 additional times with $1 \mathrm{~L}$ aliquots of $75^{\circ} \mathrm{C}$ water.

$\mathbf{1}^{\text {st }}$ Soxhlet extraction of $\mathbf{1}^{\text {st }}$ PBO fibers: A total of $10 \mathrm{~g}$ of PBO fibers ( $1^{\text {st }}$ Fiber as received) were placed in a thimble made from filter glass, which is loaded into the main chamber of the Soxhlet extractor. About $1 \mathrm{~L}$ of distilled water was used as the extraction solvent and was refluxed for $7 \mathrm{~d}$. This was done to remove all loosely bound phosphorus-containing species and additives. The fibers were tested using XRF before and after the extraction procedure to determine the amount of removed phosphorus. The extraction fluid was placed in an oven at 52 ${ }^{\circ} \mathrm{C}$ and reduced in volume to about $10 \mathrm{~mL}$. This fluid $\left(1^{\text {st }}\right.$ Water Extract of $1^{\text {st }}$ Fiber $)$ and a blank of water run through the same Soxhlet extraction and evaporation procedure were analyzed by XRF and GC/MS.

$1^{\text {st }}$ Soxhlet extraction of $2^{\text {nd }}$ and $3^{\text {rd }}$ PBO fibers: The same procedure described above was repeated with the $2^{\text {nd }}$ and $3^{\text {rd }}$ sets of PBO fibers, which were from different manufacturing lots. Samples derived from the Soxhlet procedure are labeled as follows: $2^{\text {nd }}$ Fiber as received, $1^{\text {st }}$ Water Extract of $2^{\text {nd }}$ Fiber, $1^{\text {st }}$ Extract Fiber of $2^{\text {nd }}$ Fiber, $3^{\text {rd }}$ Fiber as received, $1^{\text {st }}$ Water Extract of $3^{\text {rd }}$ Fiber, and $1^{\text {st }}$ Extract Fiber of $3^{\text {rd }}$ Fiber. 
Soxhlet extraction of PBO oligomers: Synthesized PBO oligomers were also extracted by the Soxhlet procedure described above for $14 \mathrm{~d}$ and analyzed by XRF, MALDI-TOF MS, and GC/MS.

$2^{\text {nd }}$ Soxhlet extraction of $\mathbf{1}^{\text {st }}$ PBO fibers: A 7.8 g quantity of the extracted PBO fibers $\left(1^{\text {st }}\right.$ Extract Fiber of $1^{\text {st }}$ Fiber) was placed between sheets of weighing papers (VWR Scientific Products) and then crushed with a mortar and pestle chilled using liquid nitrogen to help expose the microscopic voids found in PBO fibers (see Figure 4) to the water extractant. These crushed fibers were then extracted for $14 \mathrm{~d}$ with $800 \mathrm{~mL}$ of distilled water by the Soxhlet procedure described above. Phosphorus content in the fibers was monitored by XRF analyses before and after the $2^{\text {nd }}$ Soxhlet extraction. The final extracted fiber was labeled as $\left(2^{\text {nd }}\right.$ Extract Fiber of $1^{\text {st }}$ Fiber). The extractant was concentrated to $15 \mathrm{~mL}$. Fibers $\left(2^{\text {nd }}\right.$ Extract Fiber of $1^{\text {st }}$ Fiber $)$ and the extractant $\left(2^{\text {nd }}\right.$ Water Extract of $1^{\text {st }}$ Fiber $)$ were analyzed by MALDI-TOF MS and GC/MS to identify the species removed from the PBO fibers.

$\mathbf{1}^{\text {st }}$ Caustic treatment on $2^{\text {nd }}$ Extract Fiber of $1^{\text {st }}$ Fiber: A 3.54 g portion of $2^{\text {nd }}$ Extract Fiber of $1^{\text {st }}$ Fiber was refluxed in $1 \mathrm{~L}$ of $0.1 \mathrm{~mol} / \mathrm{L} \mathrm{NaOH}$ solution for $7 \mathrm{~d}$. After cooling down, the fibers $\left(1^{\text {st }}\right.$ Caustic treated Fiber of $1^{\text {st }}$ Fiber) were separated from the solution and dried in air. The solution ( $1^{\text {st }}$ Caustic solution of $1^{\text {st }}$ Fiber) was concentrated in the $52{ }^{\circ} \mathrm{C}$ oven to $10 \mathrm{~mL}$. XRF measurements were done before and after the $1^{\text {st }}$ Caustic treatment.

$2^{\text {nd }}$ Caustic treatment on $1^{\text {st }}$ Caustic treated Fiber of $1^{\text {st }}$ Fiber: A 3 g quantity of $1^{\text {st }}$ Caustic treated Fiber of $1^{\text {st }}$ Fiber was refluxed in $1 \mathrm{~L}$ of $1 \mathrm{~mol} / \mathrm{L} \mathrm{NaOH}$ for $7 \mathrm{~d}$. Fibers $\left(2^{\text {nd }}\right.$ Caustic treated Fiber of $1^{\text {st }}$ Fiber) were separated and dried in air. The solution was concentrated in air to about $100 \mathrm{~mL}$ then acidified with concentrated $\mathrm{HCl}$ to approximately $\mathrm{pH}$ 4. $\mathrm{NaCl}$ and $\mathrm{SiO}_{2}$ precipitated during acidification and were removed by filtration. The acidified solution was concentrated to $10 \mathrm{~mL}$. Salts precipitated during concentration were removed by filtration. Fibers before and after this caustic treatment were analyzed by XRF. The concentrated solution ( $2^{\text {nd }}$ Caustic extract solution of $1^{\text {st }}$ Fiber) was analyzed by GC/MS after methylation. 
$1^{\text {st }}$ Caustic treatment on $2^{\text {nd }}$ PBO fibers: The same caustic extraction procedure was applied to $1^{\text {st }}$ Extract Fiber of $2^{\text {nd }} P B O$ fibers. Samples were labeled $1^{\text {st }}$ Caustic extract solution of $2^{\text {nd }}$ Fiber and $1^{\text {st }}$ Caustic treated Fiber of $2^{\text {nd }}$ Fiber.

Test of Stability of Chemically Bound Non-Extractable Phosphorus: One of the two theories that have been advanced to explain the non-extractable phosphorus in PBO fibers suggests that the phosphorus is chemically bound to the polymer as aryl-phosphate esters. These arylphosphate esters in the final product are thought to arise from the known equilibrium reaction of PPA with the un-reacted hydroxyl groups of DADHB (Figure 1), with the $\mathrm{NaOH}$ neutralization and washing procedure not affecting their complete removal. Interestingly, literature results indicate that phenyl phosphates can be hydrolyzed under the caustic conditions that are normally used to wash the process fibers. Triphenyl phosphate (TPhP) and sodium phenyl phosphate dibasic dihydrate (DNaPhP) were used as model compounds to test the stability of arylphosphate esters exposed to caustic materials.

A mass of $11.3 \mathrm{mg}$ of TPhP was added to $28 \mathrm{~mL}$ of $1 \mathrm{~mol} / \mathrm{L} \mathrm{NaOH}$ solution in a single neck, $100 \mathrm{~mL}$ round-bottom flask equipped with a condenser. The mixture was refluxed for $7 \mathrm{~d}$. This mass of TPhP corresponds to $1.1 \mathrm{mg}$ phosphorus. According to XRF results, $8.9 \mathrm{mg}$ of $\mathrm{P}$ is present in $3.54 \mathrm{~g}$ of PBO fiber after two aqueous Soxhlet extractions and treatment of these fibers with a $0.1 \mathrm{~mol} / \mathrm{L} \mathrm{NaOH}$ solution. Under the test conditions, TPhP melted during boiling, but remained immiscible with water. After cooling, the contents of the flask were acidified by adding concentrated $\mathrm{HCl}$. Precipitated solids (remaining un-reacted $\mathrm{TPhP}, \mathrm{NaCl}$ and $\mathrm{SiO}_{2}$ ) were removed by filtration, and the remaining solution was concentrated to $10 \mathrm{~mL}$. Salt generated during the concentration was also removed by filtration and then extracted with diethyl ether for analysis by GC/MS. The concentrated diethyl ether extract and the concentrated solution were modified by the methylation procedure for further analysis.

The same caustic treatment procedure was performed on $7.56 \mathrm{mg}$ of sodium phenyl phosphate dibasic dihydrate (DNaPhP) in $28 \mathrm{~mL}$ of $1 \mathrm{~mol} / \mathrm{L} \mathrm{NaOH}$. DNaPhP was soluble in caustic water. The solution was acidified and concentrated to $10 \mathrm{~mL}$ after boiling for $7 \mathrm{~d}$. Solids precipitated during the procedure were removed by filtration. An aliquot of $0.5 \mathrm{~mL}$ of the concentrated solution was modified by methylation for GC/MS analysis. This procedure was 
repeated on $10.4 \mathrm{mg}$ of sodium phenyl phosphate dibasic dihydrate in $38 \mathrm{~mL}$ of $1 \mathrm{~mol} / \mathrm{L}$ methanol $\mathrm{NaOCH}_{3}$ solution.

UV-Irradiation: Ultraviolet (UV)-irradiation was performed with an apparatus equipped with a $1000 \mathrm{~W}$ xenon arc lamp (Oriel Corp). The device used in these experiments is shown in Figure 5, with the spectral output of the unit with the various attachments shown in Figure 6. Because of the exposure hazards associated with UV radiation, the device was operated according to the safety precautions in the standard operating procedure located in Appendix A.

A 4.98 g quantity of $1^{\text {st }}$ Caustic treated Fiber of $2^{\text {nd }}$ Fiber was dipped into $200 \mathrm{~mL}$ of acidified water ( $\mathrm{pH} 4)$ and divided into three portions. Each sample was placed in a wide-mouth beaker which was placed horizontally in the Oriel apparatus. Light intensity at the sample site was measured with a Newport Radiant power meter and probe, models 70260 and 70268, respectively. The light intensity at the sample was about $950 \mathrm{~W} / \mathrm{m}^{2}$. Each portion was exposed to UV radiation with no filters for $25 \mathrm{~h}$, during which the acidified water was replenished by adding $50 \mathrm{~mL}$ every $8 \mathrm{~h}$ to prevent the sample from going to dryness (open container approach). After the UV irradiation, the fibers were gathered from the beaker, squeezed to remove liquid, and dried in air. The liquid was concentrated to $21 \mathrm{~g}$, and then $1.7 \mathrm{~g}$ was methylated to facilitate GC/MS measurement.

A second procedure was performed in a sealed "quartz" chamber. The quartz container was made from a $15.56 \mathrm{~cm}(61 / 8 \mathrm{in})$ x $15.56 \mathrm{~cm}$ (6 1/8 in) x $5.08 \mathrm{~cm}$ (2 in) rectangular PMMA [i.e, poly(methyl methacrylate)] container with the two-piece top and bottom PMMA plates containing $10.16 \mathrm{~cm}$ (4 in) x $10.16 \mathrm{~cm}$ (4 in) quartz windows. The top and bottom acrylate plates were fixed to the rectangular acrylate box by removable screw bolts. In the top PMMA plate, two Teflon [i.e., poly(tetrafluoroethylene)] screw stoppers were included to facilitate the injection and extraction of liquid (see Figure 7, sealed container approach). A quantity of $3 \mathrm{~g}$ of $2^{\text {nd }}$ Caustic treated $1^{\text {st }}$ Fiber, $400 \mathrm{~mL}$ of acidified water ( $\mathrm{pH} 4$ ), and a magnetic stirring bar were put into sealed quartz chamber. The chamber was subsequently exposed to unfiltered UV radiation for $7 \mathrm{~d}$ while stirring. The fibers were removed from the chamber and filtered with a Buchner funnel utilizing Whatman filter paper. The filtrate was concentrated to $10 \mathrm{~mL}$, methylated and then analyzed by GC/MS. The amount of phosphorus in fibers before and after UV-treatment was analyzed by XRF. 
The sodium phenyl phosphate dibasic dihydrate ( $\mathrm{DNaPhP}$ ) compound was also subjected to UV irradiation. A quantity of $12.7 \mathrm{mg}$ of $\mathrm{DNaPhP}$ was put into $12.5 \mathrm{~g}$ of acidified water $(\mathrm{pH} 4)$ in a quartz container, which was then sealed with a Teflon stopper (sealed container approach). The contents were exposed to UV radiation with no filters. Aliquots of $0.5 \mathrm{~mL}$ were taken from the container after $0 \mathrm{~h}, 2 \mathrm{~h}, 12 \mathrm{~h}$ and $24 \mathrm{~h}$ for methylation and GC/MS analysis.

Methylation methods: The Soxhlet extracts were methylated using an in situ consumption diazomethane generator (see Figure 8). The generator is composed of three test tubes arranged in a stair-step fashion with test tube 1 being the highest in the arrangement. Since diazomethane presents an explosion hazard, the safety precautions listed in the standard operating procedure found in Appendix B were used. Although this approach is typically used for the methylation of carboxylic acids, diazomethane is also known to react with other compounds that have labile hydrogens such as phosphoric acid (see Figure 9) [16].

A quantity $(10 \mathrm{~mL})$ of peroxide-free diethyl ether was added to test tube 1 , and the nitrogen pressure was adjusted to give a smooth bubbling in test tube 1. A mixture of about $4 \mathrm{~mL}$ of a $37 \%$ mass fraction $\mathrm{KOH}$ solution in distilled water and $6 \mathrm{~mL}$ of carbitol was added to test tube 2 and again the nitrogen pressure was adjusted to give a smooth bubbling in test tubes 1 and 2.

Approximately $1 \mathrm{~mL}$ of each concentrated aqueous extract along with $5 \mathrm{~mL}$ of diethyl ether:ethanol (3:1) mixture was added to test tube 3. The diethyl ether:ethanol mixture was used rather than diethyl ether alone to provide a homogeneous mixture with the aqueous solution. Earlier research [17] supports this approach since diazomethane reacts with water to form methanol. Again the nitrogen pressure was adjusted to yield uniform bubbling in all three test tubes. Approximately $1.0 \mathrm{~g}$ to $1.5 \mathrm{~g}$ of diazald was added to test tube 2 . After $6 \mathrm{~min}$, additional diazald was added to test tube 2, and the reaction was continued until the sample in test tube 3 turned yellow, indicating the complete reaction of labile protons. Nitrogen was then blown over the sample in test tube 3 to evaporate residual solvents.

The solid flakes that were detected on the PBO fibers after boiling them in $\mathrm{NaOH}$ were also methylated after the following sample preparation. The solids were dissolved in an 80:20 mixture of 3:1 ether:ethanol mixture with $\mathrm{H}_{2} \mathrm{O}$. This combined mixture of ether, ethanol and water was acidified with concentrated $\mathrm{HCl}$ and methylated as described above. 


\section{Measurements:}

\section{LDI- and MALDI-TOF MS}

Mass spectra were obtained using a Bruker Daltonics (Billerica, MA) REFLEX II spectrometer. The acceleration voltage was $25 \mathrm{kV}$ and the reflectron mode was used. Delayed extraction was $0.750 \mu \mathrm{s}$. A nitrogen laser at $337 \mathrm{~nm}$ was employed as a source. External mass calibration was performed using $\mathrm{CsI}_{2}$. The resolution of the instrument is 20,000 atomic mass units (amu).

Samples were prepared by the following methods. For solid samples such as AF1, AF2, AF3, PBOm and PBO oligomer, a small quantity of sample was ground with $20 \mu \mathrm{L}$ of $\mathrm{THF} / \mathrm{HCl}$ mixture (2 mL/1 drop) using a mortar and pestle until most of the solvent evaporated. The sticky sample for doing LDI-TOF-MS was pressed using a spatula onto the stainless steel substrate which was covered by Parafilm. For liquid samples such as extracts, $1 \mathrm{~mL}$ was concentrated in an oven at $52{ }^{\circ} \mathrm{C}$. Samples were prepared using a variety of matrix/salt conditions: $\mathrm{THF} / \mathrm{HCl}$ mixture ( $2 \mathrm{~mL} / 1$ drop), acetonitrile/0.1 \% of TFA solution (1:1 by volume), and sinapinic acid in THF (10 mg/mL)/Na TFA in THF (10 mg/mL) (1:1 by volume). The prepared samples were spotted onto a stainless steel plate and air-dried (see Figure 10). From these sample preparations, a methodology was developed for identifying intractable PBO oligomers by testing the AF1, AF2, and AF3 model compounds using MALDI-TOF MS (see Figure 11 and Figure 12).

\section{GC/MS}

The GC/MS procedure for analyzing the methylated products was developed by subjecting phosphoric acid to the above methylation procedure and detecting the trimethyl ester of phosphoric acid and the associated impurities that may arise from the methylation procedures. A representative example of the total ion current trace showing the trimethyl ester of phosphoric acid and stuctures of the compounds associated with the methylation procedure are shown in Figure 13 with the identification of the associated compounds given in Figure 14.

All methylated aqueous solutions were concentrated to approximately $0.5 \mathrm{~mL}$. The methylated products were then extracted into about $3 \mathrm{~mL}$ of diethyl ether. The diethyl ether solution was then concentrated to approximately $1 \mathrm{~mL}$. These concentrated samples were then 
analyzed by GC/MS with the specific equipment being a dual injector Trace GC 2000 gas chromatograph interfaced to a Trace DSQ II mass spectrometer (MS, Thermo Finnegan - see Figure 15). The resolution of the instrument is 1,200 amu. The GC was also equipped with a flame photometric detector (FPD) and a nitrogen/phosphorus detector (NPD). The GC is equipped with a split/splitless (S/SL) injector and a programmable temperature vaporizing (PTV) injector. The effluent from a $15 \mathrm{~m}$ x $0.25 \mathrm{~mm}$ ID x $0.25 \mu \mathrm{m}$ Rxi-5MS capillary column attached to the PTV injector was split to the FPD and MS using a MS-column flow splitter (SGE Analytical Science). This device equalizes the retention times between the FPD and MS detectors, thereby allowing for phosphorus containing compounds to be distinguished in the GC/MS chromatogram. A second $15 \mathrm{~m}$ x 0.25 mm ID x $0.25 \mu \mathrm{m}$ Rxi-5MS capillary column attached to the second injector (S/SL) and connected directly to the NPD detector provides additional information about phosphorus containing compounds. Sample volumes of $1 \mu \mathrm{L}$ were injected with splitless mode. Helium was the carrier gas (flow rate: $1 \mathrm{~mL} / \mathrm{min}$ ). The oven was programmed with an initial temperature of $50^{\circ} \mathrm{C}$ and a hold time of $1 \mathrm{~min}$. The GC oven temperature was raised $20^{\circ} \mathrm{C} / \mathrm{min}$ up to $260^{\circ} \mathrm{C}$ and held at $260^{\circ} \mathrm{C}$ for $10 \mathrm{~min}$. The DSQ II mass spectrometer acquired electron impact (EI), and/or chemical ionization (CI), full scan data, with a mass range of $50 \mathrm{u}$ to $600 \mathrm{u}$, where $1 \mathrm{u}=1 \mathrm{~g} / \mathrm{mol}$. The $\mathrm{x}$-axis of the mass spectra shown in Figure 17 and Figure 18 are give in terms of $\mathrm{m} / \mathrm{z}$ (mass-to-charge) ratio. This unit is not an SI unit since the lowercase $\mathrm{m}$ is the SI unit for meter. The correct unit for mass is $\mathrm{u}$, the unified atomic mass unit. A more correct unit for use on the $\mathrm{x}$-axis for each mass spectrum would therefore be u/z. However, mass spectrometrists simply choose not to use it [18].

\section{$X R F$}

Fiber samples, liquids, and key ingredients in the synthesis and extraction experiments were analyzed by XRF for the purpose of detecting elements present and determining the approximate mass fractions of those elements other than $\mathrm{H}, \mathrm{C}, \mathrm{O}$, and $\mathrm{N}$. The materials used in the work described in this paper consisted of bunches of fibers, liquids from extraction or derivatization experiments, and various solid products from the experiments such as flakes of dried material or partially ground fibers. High-performance elemental analyses, viz. quantitative analyses, of such a variety of materials would normally be expensive and time consuming whether done by XRF or another available test method. Because the experiments in this paper 
were viewed as investigative in nature, it was decided to emphasize speed and comparability over quantitative results. This is justified because every sample required a qualitative analysis to identify elements present and a "semiquantitative" analysis to find approximately how much of each element was present. The experiments that produced the samples were expected to effect substantial changes in compositions or no changes at all.

Fundamental Parameters (FP) XRF methods are well suited to this investigative realm. FP XRF methods are designed to be interactive and are implemented as sophisticated computer programs. The investigator can specify to the computer program all a priori knowledge of the chemical and physical properties of each specimen. As an example, the PBO fibers were specified as having a chemical formula of $\mathrm{C}_{14} \mathrm{H}_{10} \mathrm{~N}_{2} \mathrm{O}_{2}$, which was chosen as the balance compound because FP XRF methods solve a set of equations with the SUM = $100 \%$ constraint. Similarly, aqueous solutions were specified with $\mathrm{H}_{2} \mathrm{O}$ as the balance compound, and white flakes found in some fibers were specified with $\mathrm{C}_{6} \mathrm{H}_{10} \mathrm{O}_{5}$ (a general formula for cellulose from filter paper) as the balance compound. In some cases, the elements of interest were specified as oxides, when there was evidence that additional oxygen was present beyond the PBO compounds. However, in nearly all cases, the inorganic elements in the fibers were simply calculated as the elements.

The FP XRF program used in this work was the IQ+ method from PANalytical, Inc (Almelo, The Netherlands), which was run in the SuperQ operating system, version 4.0d, of a model PW2404 wavelength-dispersive spectrometer, also from PANalytical, Inc. The IQ+ method was calibrated using a set of glass and briquette standards provided by the vendor, which was bolstered by a number of NIST Standard Reference Materials. This calibration scales the calculations to the actual performance of the spectrometer employed in this effort. The program was originally calibrated in 2001 and has been maintained by implementing drift correction updates since that time. All measurements were made in scanning mode, in vacuum for solids or He for liquids, with the Rh anode X-ray tube operated at $3 \mathrm{~kW}$. As was mentioned, physical properties of the specimens may be included to help set boundary conditions for the FP calculations. All specimens were weighed into sample cells, and an estimate of the viewed area was made. These values were entered into the program to help scale the expected X-ray count rates calculated from the FP equations. The cells were $25 \mathrm{~mm}$ polyethylene (Somar, Las Vegas, $\mathrm{NV}$ ) with $6 \mathrm{~mm}$ polypropylene window material. 
The performance of FP XRF methods such as IQ+ can best be described as "semiquantitative" in that it varies from quantitative with well-controlled specimens to 'order of magnitude' results, when specimens are difficult to handle, of very small quantity, or have some other complicating properties. The PBO fiber specimens are of moderate difficulty to analyze because they are a challenge to present to the spectrometer for measurements. XRF methods are at their best when all specimens are of the same physical characteristics in that they have the same size, shape, and mass, and when all of their constituent elements can be measured and directly calculated as mass fractions summing to $100 \%$. PBO fibers, powders, water solutions, and the like do not conform to this ideal situation. The fibers cannot be positioned inside cells in a perfectly repeatable manner because they do not lay flat on the inside bottom of the cell. Water and organic materials are mostly $\mathrm{C}, \mathrm{N}$, and $\mathrm{O}$, which cannot be measured when the specimen is placed inside a cup-shaped cell. The fluorescent $\mathrm{X}$ rays from these elements cannot penetrate the thin polymer window supporting the specimen at the bottom of the cell.

The uncertainty of XRF results was evaluated by analyzing specimens of SRM 1575a Pine Needles, which contains 0.107 \% total P. Measured results for P in SRM 1575a ranged from quantitative when sufficient sample mass was provided to biased low by approximately $35 \%$ when the sample mass was only $20 \mathrm{mg}$. The results for other elements present in SRM 1575a were observed to be biased either high or low by amounts ranging from $50 \%$ to a factor of 10 depending on the mass of sample, the element, and the energy of the X-ray line measured for that element. On the Certificate of Analysis, it is recommended to use at least $250 \mathrm{mg}$ of SRM 1575a for a determination. In addition, quantities $<100 \mathrm{mg}$ did not cover the entire viewed area of the specimen cup, and results were low because $\mathrm{P} K-\mathrm{L}_{2,3} \mathrm{X}$ rays are of low energy and do not penetrate deeply into most materials. Therefore, samples of low mass and uneven distribution in a cell are expected to yield biased results. This source of bias is expected to affect the PBO and other samples studied in this work. The results for P reported in Table 1 have been assessed an overall relative uncertainty based on a non-symmetrical, triangular distribution of + $10 \%$ to - $50 \%$ relative (approximate $95 \%$ level of confidence) based on the observed performance for SRM 1575a. However, comparisons of values in Table 1 should be viewed in the context of the expected repeatability of measurements for the IQ+ method. Based on past performance of the method, the relative standard deviation for repeatability is approximately 
$10 \%$. The additional elements reported in Table 1 are provided for forensic purposes. For each element, differences of a factor of two or greater may be significant.

\section{RESULTS AND DISCUSSIONS}

Based on the nomenclature in prior accounts [4,9,10], the results will be discussed in terms of extractable and non-extractable phosphorus in PBO fibers. Extractable phosphorus is defined as the phosphorus removed during the water Soxhlet extraction procedure, while nonextractable phosphorus is defined as requiring mechanical or chemical means to remove it from the fiber.

\subsection{Extractable Phosphorus in PBO Fibers}

With recognition of the possible presence of phosphorus-containing processing additives in manufactured PBO fibers, the Soxhlet extraction method was employed to separate free acid species from non-extractable phosphorus. Table 1 shows XRF results for the PBO fibers before and after Soxhlet extraction and the extracted solutions. Consistent with previous research, the $1^{\text {st }}$ set of PBO fibers were found to contain about $0.38 \%$ phosphorous by mass. After Soxhlet extraction for $7 \mathrm{~d}$, data from the $1^{\text {st }}$ set of PBO fibers indicates that $75 \%$ of the phosphorous remained in the fibers. The phosphorus level in the $1^{\text {st }}$ set of PBO fibers is consistent with PBO fiber analyses from previous research $[9,10]$. Additionally, the amount removed by Soxhlet extraction is consistent with the quantity removed by super critical fluid extraction [9]. Comparatively, the initial phosphorus levels in the $2^{\text {nd }}$ and $3^{\text {rd }}$ sets of PBO fibers were $16 \%$ and $37 \%$ lower, respectively, than the $1^{\text {st }}$ set of PBO fibers. These reductions may reflect inconsistencies in the $\mathrm{NaOH}$ washing procedure during manufacturing or a change by the manufacturer to a more efficient washing process. Consistent with the $1^{\text {st }}$ set of PBO fibers, the Soxhlet extraction procedure removed little or none of the residual phosphorus from the as received samples. Furthermore, a subsequent Soxhlet extraction of the $1^{\text {st }}$ set of PBO fibers after they were damaged by crushing caused no additional reduction in the level of phosphorus, further suggesting that the remaining phosphorus is not extractable.

In the $1^{\text {st }}$ set of PBO fibers, the aqueous extract was concentrated and acidified with $\mathrm{HCl}$ to convert any extracted acid salts to the free acid. An attempt was made to extract these 
phosphorus species into diethyl ether for the methylation procedure with no success. For the $2^{\text {nd }}$ and $3^{\text {rd }}$ PBO fiber samples, the extracted phosphorus containing species were also concentrated as describe in the Experimental Section and acidified with $\mathrm{HCl}$. In a departure from the procedure used on the extractant from the $1^{\text {st }}$ set of PBO fibers, a $1 \mathrm{~mL}$ portion of the concentrated extract was mixed with a 3:1 diethyl ether:ethanol mixture, methylated as described in the experimental section, and analyzed by GC/MS under electron impact (EI) and chemical ionization (CI) test conditions along with FPD and NPD detection.

The total ion current (TIC) chromatograms and the parallel FPD chromatograms are shown in Figure 16 for the samples run under EI and CI test conditions. In the FPD chromatograms (Figure 16(b) and Figure 16(d)), two peaks at retention times of 4.12 min and 14.16 min under EI conditions and $3.77 \mathrm{~min}$ and $13.42 \mathrm{~min}$ under CI conditions are shown that indicate phosphorus containing compounds. The EI and CI mass spectra of the first peak are shown in Figure 17(a) and Figure 17(b). From Figure 17(b), the characteristic ions parent+1 u, parent $+29 \mathrm{u}$, and parent $+41 \mathrm{u}$ observed under CI conditions indicate that the molecular mass of the first eluting phosphorus containing species is $140 \mathrm{u}$. The EI spectrum, which is consistent with the published spectrum of the trimethyl ester of phosphoric acid (CAS \# 512-56-1) [19,20], indicates the successive loss of two units of $30 \mathrm{u}$ from the parent ion $(140 \mathrm{u})$ and the parent- $1 \mathrm{u}$ ion to form the fragment ions observed at $110 \mathrm{u}$ and $80 \mathrm{u}$ and $109 \mathrm{u}$ and $79 \mathrm{u}$, respectively. The losses from the parent ion have been shown to be exclusively linked to the elimination of the neutral species $\mathrm{CH}_{2} \mathrm{O}$ [20]. Therefore, the EI and CI spectra of the first eluting peak indicate the presence of extractable phosphoric acid from the PBO fibers.

From Figure 17(d), ions at 379 u, $407 \mathrm{u}$, and $419 \mathrm{u}$ in the second eluted peak correspond to the characteristic ions mentioned above for CI molecular ion determination and indicate that the molecular mass of this species is $378 \mathrm{u}$. The absence of the corresponding molecular ion under EI conditions (Figure 17(c)) and the indication from Figure 16(b) and Figure 16(d) that this compound contains phosphorus suggest that this species may be a phosphate ester with a long alkyl chain. The dominant fragment ion at $127 \mathrm{u}$ can be assigned to the structure shown in Figure 17(c), further supporting the identification of this species as a long alkyl chain, dimethylester phosphate, where the dimethyl ester groups on this trialkyl phosphate ester arise from the methylation procedure. 
It is known that ethyl and higher alkyl phosphate esters undergo the classic McLafferty + 1 rearrangement to eliminate the alkyl groups as a radical from the molecular ion [21]. Furthermore, the EI mass spectrum of the diethyl pentyl ester of phosphoric acid (DEPEPA, relative molecular mass = 224 u, CAS \# 20195-08-8) contains the corresponding rearrangement fragment ion at $155 \mathrm{u}$, which reflects the presence of the diethyl groups rather than the dimethyl groups indicated on the $127 \mathrm{u}$ fragment ion found in Figure 17(c). Successive losses of $28 \mathrm{u}$ below the $155 \mathrm{u}$ fragment ion are observed in DEPEPA that probably correspond to successive losses of the neutral species $\mathrm{C}_{2} \mathrm{H}_{4}$. These losses are not observed in Figure 17(c). Finally, the published spectra of the trimethyl silyl (TMS) derivative of 1-dodecyl- $\mathrm{d}_{25}$ phosphate also exhibits the characteristic McLafferty +1 rearrangement to eliminate the deuterated dodecyl alkyl group as a radical from the molecular ion (see Figure 18) [22]. From these observations, the second phosphate compound seems to be an octadecyl dimethyl ester of phosphoric acid. This identification indicates that octadecyl phosphate or the disodium salt of octadecyl phosphate is being used as a processing aid by the manufacturer.

It is worth noting that even though the semi-quantitative XRF analyses of the $2^{\text {nd }}$ and $3^{\text {rd }}$ sets of PBO fibers before and after Soxhlet extraction showed little or no discernible differences in phosphorus levels, the extractant from the $2^{\text {nd }}$ set of PBO fibers showed by GC/MS, one of the most sensitive analytical tools for detecting unknown compounds, the presence of extractable phosphoric acid and n-octadecyl phosphate. This indicates that in these latter sets of PBO fibers, the majority of the residual phosphorus is non-extractable.

\subsection{Non-Extractable Phosphorus in PBO Fibers}

Two theories have been put forth to explain the presence and nature of the nonextractable phosphorus. The first theory suggests that phosphoric acid may be trapped in microscopic voids that are known to exist along the length of the PBO fiber (see Figure 4). In the presence of moisture, the trapped phosphoric acid in these microscopic voids is suspected of lowering the localized $\mathrm{pH}$ in the PBO material surrounding them, thereby creating a localized environment conducive to hydrolytic degradation. The second theory suggests that the residual phosphoric acid, if present, may be chemically bound to the PBO polymer chain structure as a monoaryl phosphate ester (see Figure 19). 
To address the first theory a second extraction was performed on the $1^{\text {st }}$ set of PBO fibers that had been previously extracted with water by the Soxhlet procedure. These fibers were cut into small pieces and ground with a mortar and pestle cooled by liquid nitrogen in an attempt to disrupt the microscopic voids and make them more accessible to moisture. Although a semiquantitative assessment of the damage caused by grinding the fibers was not obtained, XRF results for the cut and ground fibers did not show a decrease of the amount of phosphorous compared to the analysis after the first extraction (see Table 1). Consistent with these results, XRF results for the $2^{\text {nd }}$ extracted solution from the $1^{\text {st }}$ set of PBO fibers indicated the presence of phosphorous comparable to that of the water blank. This result suggests that either the fibers were not sufficiently damaged by the crushing procedure or phosphoric acid is not trapped in the microvoids.

To pursue the second theory, the twice extracted fibers from the $1^{\text {st }}$ set of PBO fibers were boiled for $7 \mathrm{~d}$ in $0.1 \mathrm{~mol} / \mathrm{L} \mathrm{NaOH}$ caustic solution. A small decrease in the phosphorus level was observed by XRF. The boiling experiment was repeated with $1 \mathrm{~mol} / \mathrm{L} \mathrm{NaOH}$, with no observable change in phosphorus levels. To verify these results, the $2^{\text {nd }}$ set of PBO fibers was boiled with $1 \mathrm{~mol} / \mathrm{L} \mathrm{NaOH}$. Consistent with the $1^{\text {st }}$ set of PBO fibers the phosphorus level decreased slightly from $0.29 \%$ mass fraction to $0.27 \%$ mass fraction (see Table 1). A recheck of the $1^{\text {st }}$ set of PBO fibers after boiling in $\mathrm{NaOH}$ revealed the presence of solid flakes in these fibers. Reanalysis of the twice Soxhlet extracted and twice $\mathrm{NaOH}$ boiled $1^{\text {st }}$ set of fibers after removal of the flakes showed them to exhibit no change or a slight increase in the phosphorus level. The amount is also comparable to that found in the $2^{\text {nd }}$ set of PBO fibers after Soxhlet extraction and treatment with $1 \mathrm{~mol} / \mathrm{L} \mathrm{NaOH}$.

The solid flakes were found to contain sodium, silicon, with some phosphorus. After acidifying the solids and methylating the resulting aqueous solution, octadecyl phosphate, with no indication of phosphoric acid, was detected. The presence of this processing additive after boiling in the $\mathrm{NaOH}$, may reflect the increased solubility of disodium octadecyl phosphate in water relative to octadecyl phosphate. It should be noted that the basic solutions from these $\mathrm{NAOH}$ treatments did not indicate the presence of phosphorus by XRF and after they were concentrated, acidified, and methylated for analysis by GC/MS. Therefore, the $\mathrm{NaOH}$ treatments appear to slightly lower the level of phosphorus in the fibers that have been previously subjected 
to the Soxhlet extraction procedure by converting the remaining octadecyl phosphate, which has limited solubility in water, to the more water soluble disodium salt.

In a parallel investigation, the phosphorus level in low-mass average molecular mass model PBO oligomers were investigated with the goal being to look for the presence of phosphorus containing species in these intractable model oligomers by MALDI-TOF MS. After extensive rinsing of the model PBO oligomers with water, the final phosphorus level by XRF was $1.4 \%$ (see Table 1). Soxhlet extraction of these oligomers reduced the phosphorus level to $0.062 \%$ mass fraction, with GC/MS identifying the phosphorus species in the aqueous extract as phosphoric acid detected as the trimethyl ester (see Figure 20). Boiling these extracted oligomers $0.1 \mathrm{~mol} / \mathrm{L} \mathrm{NaOH}$ for $7 \mathrm{~d}$ reduced the phosphorus level to $0.036 \%$ mass fraction. Again, the removed phosphorus species in the $\mathrm{NaOH}$ solution was identified as phosphoric acid.

PBO oligomers, due to the limited solubility of TA in the reaction medium, are preferentially capped with DADHB during the manufacturing process (see Figure 1). However, in the model compound studies performed by So et al. [23, see compounds (3) and (5)] these researchers detected the presence of 4-(6-[4-(2-benzoxazolyl)phenyl]benzo(1,2-d;5,4-d')bisoxazol-2-yl) benzoic acid and 4-(2-benzoxazolyl)-benzoic acid when their model compounds were dissolved in methanesulfonic acid. These species are known to exist in the PPA reaction medium as aryl-acetyl PPA esters (see Figure 1) and can remain after the neutralization procedure as aryl-acetyl phosphate esters. These types of esters are known to be stable under neutral conditions, while undergoing hydrolysis under basic conditions [24]. Therefore, it is probable that the additional phosphoric acid removed from our model systems by treatment with $\mathrm{NaOH}$ came from aryl-acetyl phosphate esters that are not typically found in processed PBO fibers. Some support for the supposition can be found in Figure 20 where the methyl ester of TA was detected as being extracted from the PBO model oligomers.

Analysis of the low-molecular mass PBO oligomers, synthesized as described in the experimental section, showed the presence of a peak at $688.5 \mathrm{u}$ (see Figure 21). This compound was identified as being a mono-PBO phosphate ester (an aryl-phosphate) that differs structurally from the aryl-acetyl phosphate ester discussed above. The other compounds identified from peaks in Figure 21 are shown in Figure 22. Reanalysis of the solids by MALDI-TOF MS showed that the compound at $688.5 \mathrm{u}$ was not removed by Soxhlet extraction. Interestingly, this 
compound was not removed by boiling the solid oligomers with $0.1 \mathrm{~mol} / \mathrm{L} \mathrm{NaOH}$ caustic solution.

Noting the consistency of the model PBO oligomer results with those of the PBO fibers, the apparent stability of at least some of the non-extractable phosphorus to $\mathrm{NaOH}$ treatment is surprising since the working hypothesis considers the phosphorus to be chemically bound in the PBO oligomers as phosphate esters, and phosphate esters can be hydrolyzed under alkaline conditions [25]. To investigate this further, the model compound TPhP (see Figure 19) was refluxed in a $1 \mathrm{~mol} / \mathrm{L} \mathrm{NaOH}$ solution for $7 \mathrm{~d}$. After the solution was concentrated and methylated, $95 \%$ of the converted TPhP was found to be in the form of the dimethyl ester of monophenyl phosphate (DMPhP), with only 4.3 \% being converted to TMP (see Table 2).

To verify this surprisingly low conversion of TPhP to TMP, sodium phenyl phosphate dibasic dihydrate (DNaPhP) (see Figure 19) was refluxed in a $1 \mathrm{~mol} / \mathrm{L} \mathrm{NaOH}$ solution for $7 \mathrm{~d}$. The conversion from DNaPhP to the trisodium phosphate salt (TNaP), as reflected in the methylated products of TMP and DMPhP by GC/MS, was less than $2 \%$. A similar experiment in a $1 \mathrm{~mol} / \mathrm{L} \mathrm{NaOCH}_{3}$ solution resulted in a slightly higher conversion of $6.9 \%$ of $\mathrm{DNaPhP}$ to TMP (see Table 2). The results indicate that if the non-extractable phosphorus is chemically bound to the PBO polymer as the monoaryl phosphate ester (see Figure 19), it may be partially stable to the $\mathrm{NaOH}$ solutions that are commercially used to effect its removal.

From the above results, sodium phenyl phosphate dibasic dihydrate (DNaPhP) was converted to the acid, monophenyl phosphate, using an $\mathrm{HCl}$ solution with a final $\mathrm{pH}$ of 4 . This solution was then placed in a sealed container and irradiated with UV light (sealed chamber approach). The conversion of monophenyl phosphate to phosphoric acid, as measured by the presence of TMP by GC/MS, was completed within $24 \mathrm{~h}$ (see Table 2). These results indicate that if the non-extractable phosphate is in the form of aryl-phosphate esters it may be removed by placing the fibers in an acidic medium and exposing it to UV radiation. The $1^{\text {st }}$ set of PBO fibers that had been Soxhlet extracted twice and washed treated with $0.1 \mathrm{~mol} / \mathrm{L}$ and $1 \mathrm{~mol} / \mathrm{L} \mathrm{NaOH}$ solutions for $7 \mathrm{~d}$ each was washed with $\mathrm{pH} 4$ aqueous solution that was acidified with $\mathrm{HCl}$. The washed fibers were divided into three portions. Each portion was placed in an aqueous medium acidified to $\mathrm{pH} 4$ by $\mathrm{HCl}$ and irradiated with $\mathrm{UV}$ for $25 \mathrm{~h}$. The acid medium was replenished as needed to keep the sample from becoming dry (open chamber approach). The acid mediums were combined, concentrated and tested for TMP, with very little being detected. 
Observing that the experiment on the $\mathrm{DNaPhP}$ model compound was performed in a closed system where the $\mathrm{HCl}$ could not escape, the $2^{\text {nd }}$ set of PBO fibers that had been Soxhlet extracted and treated with $1 \mathrm{~mol} / \mathrm{L} \mathrm{NaOH}$ solutions for $7 \mathrm{~d}$ each was treated with $\mathrm{pH} 4$ aqueous solution as in the above procedure but this time the fibers and acidic solution were placed in a sealed system that has been described in the experimental section. GC/MS analysis of the aqueous solution indicated the presence of significant amounts of phosphoric acid in the form of trimethylphosphate (see Figure 23). It must be noted that the companion XRF analysis (see Table 1) also indicated a decrease in the phosphorus level of the acid treated and UV exposed fibers by the closed system.

\section{CONCLUSIONS}

The data presented herein indicates that some free phosphoric acid is present in PBO fibers with a significant portion of the extractable phosphorus being n-octadecyl phosphate (either as the diacid or the diacid salt) that had been added as a processing aid. This result underscores the need to go beyond elemental analysis and determine the chemical identity of the residual phosphorus in the PBO fibers. The slight to moderate decrease in the residual phosphorus levels between the $1^{\text {st }}$ set of PBO fibers and the $2^{\text {nd }}$ and $3^{\text {rd }}$ sets of PBO fibers suggests a possible change in the manufacturing process to better remove readily extractable phosphorus from the PBO fibers. With this potential reduction in residual phosphorus, the $2^{\text {nd }}$ and $3^{\text {rd }}$ PBO fiber sets indicate that the primary remaining phosphorus is chemically bound to the PBO oligomers, possibly in the form of monoaryl phosphate esters that are resistant to effective removal by washing with $\mathrm{NaOH}$. MALDI-TOF MS of low mass average molecular mass PBO oligomers synthesized in a manner consistent with the manufacturing process supports the presence of monoaryl phosphate esters in PBO fibers. Subsequent studies of triphenyl phosphate and monophenyl phosphate also support the stability of monoaryl phosphates to $\mathrm{NaOH}$ treatments. However, acid treatments of monophenyl phosphate showed complete conversion to phosphoric acid. Consistent with this latter result, acid treatment of PBO fibers that had been previously Soxhlet extracted and $\mathrm{NaOH}$ treated showed that additional phosphorus could be removed from the fibers in the form of phosphoric acid in a closed system that minimizes the loss of $\mathrm{HCl}$. Interestingly, So et al. [7] deduced the presence of a monoaryl phosphate ester 
attached to PBO oligomers from their model compound studies using NMR. Consistent with the findings in this report, they observed that by placing one drop of water on the sample, the phosphate they deduced in the model compound studies as being in the form of a monoaryl phosphate slowly hydrolyzed under acid conditions. 


\section{REFERENCES AND NOTES}

1. H. Vogel; C. S. Marvel, J Polym Sci, 50, 511 (1961)

2. H. Vogel; C. S. Marvel, J Polym Sci A1, 34, 1125 (1996)

3. P. M. Cunniff; M. A. Auerbach; E. Vetter; D. J. Sikkema, "23rd. Army Science Conference", Assistant Secretary of the Army (Acquisition, Logistics and Technology), Orlando, Florida, (Dec. 2002),

4. D.E. McLemore, Interim Summary of Finding on Toyobo's Zylon Fiber; Addressee: D. E. Wagner, Esq; July 202005.

5. P. J. Walsh; X. Hu; P. M. Cunniff; A. J. Lesser, J Appl Polym Sci, 102, 3517 (2006)

6. Y.-H. So; J. P. Heeschen, J Org Chem, 62, 3552 (1997)

7. Y.-H. So; J. P. Heeschen; B. Bell; P. Bonk; M. Briggs; R. DeCaire, Macromolecules, 31, 5229 (1998)

8. G. A. Holmes; K. Rice; C. R. Snyder, J Mater Sci, 41, 4105 (2006)

9. X. Hu; A. J. Lesser Post-Treatment of Poly-p-Phenylenebenzobisoxazole (PBO) Fibers Using Supercritical Carbon Dioxide.; 2004.

10. J. Chin; E. Byrd; A. Forster; X. Gu; T. Nguyen; W. Rossiter; S. Scierka; L. Sung; P. Stutzman; J. Sieber; K. Rice, Chemical and Physical Characterization of Poly(p-Phenylene Benzobisoxazole) Fibers Used in Body Armor; NISTIR 7237; National Institute of Standards and Technology, Gaithersburg, MD 20899, Mar. 2005.

11. P. F. Jackson; K. J. Morgan; A. M. Turner, J Chem Soc Perk T 2, 11, 1582 (1972)

12. Sen, A., Tani, K., and Katoh, K., U.S. Patent 5393478

13. P. J. Walsh; X. Hu; P. M. Cunniff; A. J. Lesser, J Appl Polym Sci, 102, 3819 (2006)

14. J. F. Wolfe; F. E. Arnold, Macromolecules, 14, 909 (1981)

15. T. Kitagawa; H. Murase; K. Yabuki, J Polym Sci Pol Chem, 36, 39 (1998)

16. D. Z. Brunengraber; B. J. McCabe; J. Katanik; S. F. Previs, Anal Biochem, 306, 278 (2002)

17. A. Bhati, J Chem Soc, 729 (1963)

18. K. L. Busch, Spectroscopy, 16, 28 (2001)

19. Heller, S. R. and Milne, G. W. A., "EPA/NIH Mass Spectral Data Base", U.S. Government Printing Office: Washington, D.C., 1 (Molecular Weights 30-186) (1978) 1-987. 
20. D. A. Bafus; E. J. Gallegos; R. W. Kiser, The Journal of Physical Chemistry, 70, 2614 (1966)

21. F. W. McLafferty, Analytical Chemistry, 31, 82 (1959)

22. L. T. Sniegoski; E. White, Journal of Labelled Compounds and Radiopharmaceuticals, 20, 303 (1983)

23. Y.-H. So; S. J. Martin; K. Owen; P. B. Smith; C. L. Karas, J Polym Sci A1, 37, 2637 (1999)

24. L. L. Cameron; S. C. Wang; R. Kluger, JACS, 126, 10721 (2004)

25. T. S. Whitesides; S. H. Hilal; L. A. Carreira, QSAR Comb Sci, 25, 123 (2006) 
Table 1. Selected X-Ray Fluorescence Results for PBO Fibers, Model Oligomers and Liquids (All values are mass fraction (\%)).

\begin{tabular}{|c|c|c|c|c|c|c|c|c|c|}
\hline \multirow[b]{2}{*}{ Fiber Samples } & \multicolumn{9}{|c|}{ ELEMENTS } \\
\hline & $\mathrm{Na}$ & $\mathrm{Al}$ & $\mathrm{Si}$ & $\mathrm{P}$ & $\mathrm{s}$ & $\mathrm{Cl}$ & $\mathrm{K}$ & $\mathrm{Ca}$ & $\mathrm{Fe}$ \\
\hline \multicolumn{10}{|l|}{$1^{\text {st }}$ Set of Fibers } \\
\hline PBO Fibers as received & 0.31 & 0.002 & 0.007 & 0.38 & 0.004 & 0.047 & 0.012 & 0.040 & \\
\hline PBO Fibers after water wash & 0.19 & 0.005 & 0.022 & 0.28 & 0.003 & 0.008 & 0.010 & 0.005 & 0.002 \\
\hline PBO damaged (by grinding) & 0.20 & 0.013 & 0.009 & 0.30 & 0.004 & 0.007 & 0.010 & 0.009 & 0.029 \\
\hline PBO treated with $0.10 \mathrm{~mol} / \mathrm{L} \mathrm{NaOH}$ & 8.6 & 0.033 & 1.7 & 0.25 & 0.002 & 0.009 & & 0.006 & 0.006 \\
\hline PBO treated with $1.0 \mathrm{~mol} / \mathrm{L} \mathrm{NaOH}$ & 0.45 & 0.065 & 0.49 & 0.25 & & 0.008 & & 0.029 & 0.017 \\
\hline Flakes from $\mathrm{NaOH}$ treated fibers & 1.8 & 0.14 & 18 & 0.020 & & 2.0 & & 0.010 & 0.040 \\
\hline Fibers after removing flakes & 0.58 & 0.038 & 0.71 & 0.28 & 0.008 & 0.012 & 0.010 & 0.024 & 0.005 \\
\hline Fibers after UV irradiation (open) & 0.16 & 0.005 & 0.20 & 0.26 & & 0.023 & & 0.002 & 0.004 \\
\hline \multicolumn{10}{|l|}{$2^{\text {nd }}$ Set of Fibers } \\
\hline PBO Fibers as received & 0.20 & & 0.004 & 0.32 & 0.001 & 0.014 & 0.040 & 0.005 & 0.033 \\
\hline PBO Fibers after water wash & 0.12 & 0.009 & 0.012 & 0.29 & 0.002 & 0.011 & & 0.013 & 0.035 \\
\hline PBO treated with $1.0 \mathrm{~mol} / \mathrm{L} \mathrm{NaOH}$ & 0.45 & 0.035 & 0.34 & 0.27 & 0.003 & 0.014 & & 0.009 & 0.007 \\
\hline Fiber after UV irradiation (closed) & 0.052 & 0.025 & 0.69 & 0.21 & & 0.064 & & & 0.002 \\
\hline \multicolumn{10}{|l|}{$3^{\text {rd }}$ Set of Fibers } \\
\hline PBO Fibers as received & 0.15 & & 0.005 & 0.24 & 0.003 & 0.008 & 0.024 & 0.003 & 0.002 \\
\hline PBO Fibers after water wash & 0.20 & & 0.003 & 0.25 & 0.001 & 0.007 & & 0.003 & 0.021 \\
\hline \multicolumn{10}{|l|}{ PBO Model Oligomers } \\
\hline Before extraction & & 0.008 & 0.016 & 1.4 & & 0.027 & & 0.010 & 0.013 \\
\hline After extraction & & 0.003 & 0.004 & 0.062 & 0.001 & 0.12 & & 0.002 & 0.013 \\
\hline Treated $0.10 \mathrm{~mol} / \mathrm{L} \mathrm{NaOH}$ & 0.61 & 0.020 & 0.12 & 0.036 & 0.001 & 0.10 & & 0.19 & 0.034 \\
\hline \multicolumn{10}{|l|}{ Liquid Samples } \\
\hline Blank solvent & 0.043 & 0.009 & 0.27 & 0.024 & 0.001 & & & 0.010 & \\
\hline Extract from damaged fibers & 0.034 & 0.021 & 0.50 & 0.026 & Trace & & 0.006 & 0.002 & \\
\hline Extract from PBO model oligomers & 0.038 & & 0.16 & 0.80 & & & & 0.010 & \\
\hline
\end{tabular}

\section{COMMENTS}

Uncertainty: For P, the uncertainty (approx $95 \%$ confidence level) is + $10 \%$ to $-50 \%$.

For other elements, differences $>100 \%$ may be significant.

PBO polymer fibers were modeled as $\mathrm{C}_{14} \mathrm{H}_{10} \mathrm{~N}_{2} \mathrm{O}_{2}$.

Liquid samples were modeled as $\mathrm{H}_{2} \mathrm{O}$ with $\mathrm{P}$ as $\mathrm{PO}_{4}$ and $\mathrm{S}$ as $\mathrm{SO}_{3}$.

Flakes were modeled as cellulose $\mathrm{C}_{6} \mathrm{H}_{10} \mathrm{O}_{5}$ because they contain weighing paper.

A blank cell means the element was not detected.

A note 'trace' indicates the element was detected, but was $<0.001 \%$ 
Table 2. Conversion of Triphenyl phosphate and Monophenyl phosphate to Phosphoric Acid in Caustic Solutions and Acid Solution plus UV Exposure

\begin{tabular}{|c|c|c|c|c|c|}
\hline \multirow[t]{2}{*}{ Samples } & \multirow[t]{2}{*}{ Conditions } & \multicolumn{3}{|c|}{ Water solution } & \multirow{2}{*}{$\begin{array}{l}\text { Solid } \\
\mathrm{TPhP}\end{array}$} \\
\hline & & TMP & DMP & MMP & \\
\hline \multirow[t]{2}{*}{ Triphenyl phosphate (TPhP) } & strong caustic treat $(1 \mathrm{~N} \mathrm{NaOH})$ & $3.4 \%$ & $74.5 \%$ & $0.2 \%$ & $21.9 \%{ }^{*}$ \\
\hline & & $4.3 \%^{+}$ & $95.4 \%^{+}$ & $0.3 \%^{+}$ & \\
\hline \multirow{8}{*}{$\begin{array}{l}\text { Monophenyl phosphate, } \\
\text { dibasic sodium, dihydrate (MPhP) }\end{array}$} & & TMP & $\mathrm{MPhP}$ & & \\
\hline & strong caustic treat $(1 \mathrm{~N} \mathrm{NaOH})$ & $1.80 \%$ & $98.20 \%$ & & \\
\hline & strong caustic treat $\left(1 \mathrm{~N} \mathrm{NaOCH}_{3}\right)$ & $6.90 \%$ & $93.10 \%$ & & \\
\hline & & TMP & $\mathrm{MPhP}$ & & \\
\hline & UV-treat $0 \mathrm{~h}$ & $0.2 \%$ & $99.8 \%$ & & \\
\hline & UV-treat $2 \mathrm{~h}$ & $16.1 \%$ & $83.9 \%$ & & \\
\hline & UV-treat $12 \mathrm{~h}$ & $73.4 \%$ & $26.6 \%$ & & \\
\hline & UV-treat $24 \mathrm{~h}$ & $99.8 \%$ & $0.2 \%$ & & \\
\hline
\end{tabular}

${ }^{*}$ Measured with ether extract from solid

${ }^{+}$Recalculated composition only in water extract. 

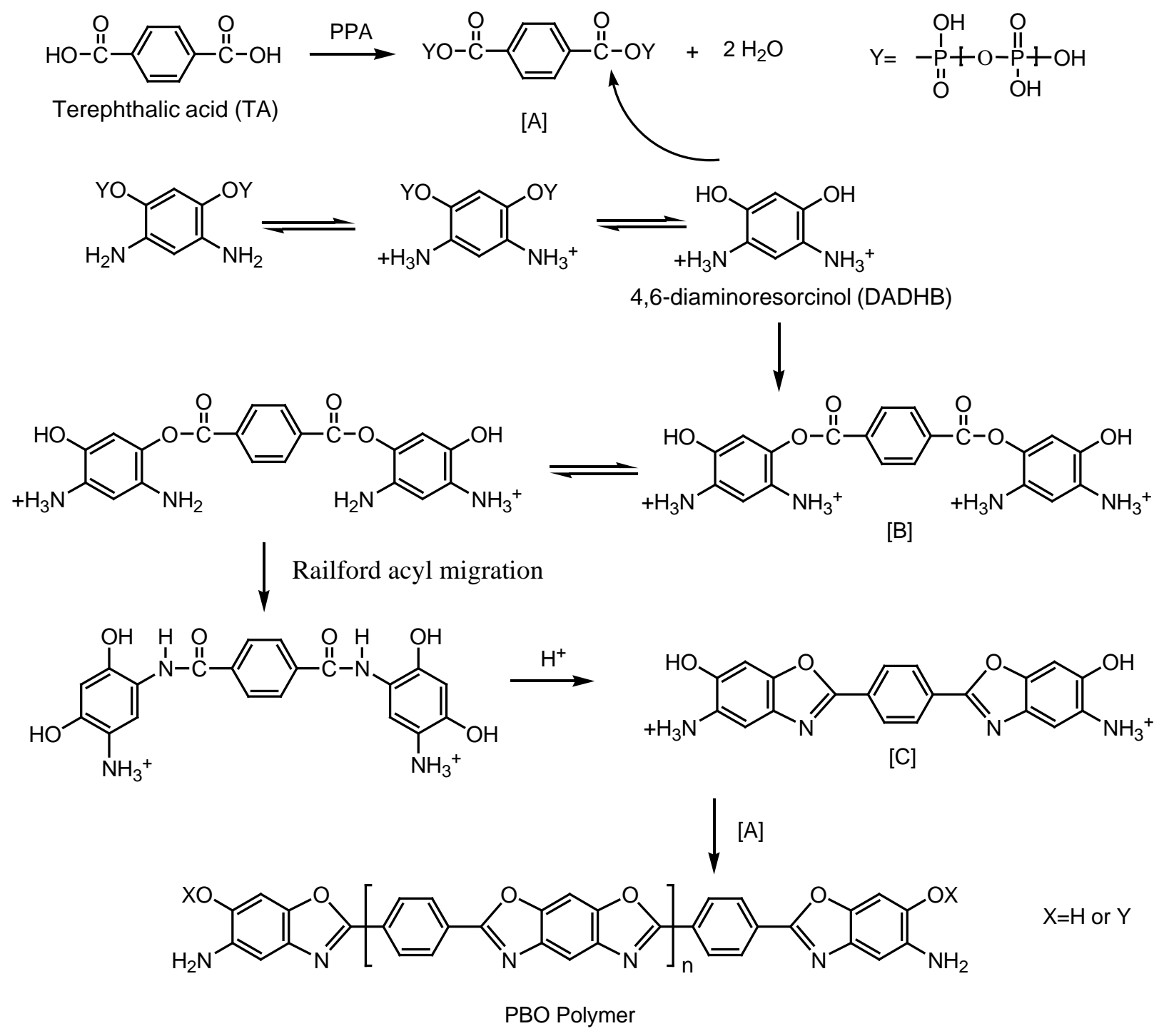

Figure 1. Reaction scheme for the preparation of PBO polymer (adapted from So et al. [6,7]). 


\section{Model Compounds for Studying Hydrolytic and UV Stability of PBO Chain Structure}<smiles>c1ccc(-c2nc3cc4nc(-c5ccccc5)oc4cc3o2)cc1</smiles>

AF2

2,6-Diphenylbenzo[1,2-d;5,4-d']bisoxazole 2 Benzoic acid + DADHB Dihydrochoride molecular mass $=312.33 \mathrm{~g} / \mathrm{mol}$ (Amines not conjugated)<smiles>c1ccc2oc(-c3ccc(-c4nc5ccccc5o4)cc3)nc2c1</smiles>

AF1 2,2'-(1,4-Phenylene)bis(benzoxazolyl) 2 (2-AP) Hydrochloride + TA molecular mass $=312.33 \mathrm{~g} / \mathrm{mol}$ (Amines conjugated)<smiles>c1ccc(-c2nc3ccccc3o2)cc1</smiles>

\section{AF3}

2-Phenylbenzoxazole

2-AP Hydrochloride + Benzoic acid molecular mass $=195.2 \mathrm{~g} / \mathrm{mol}$

Figure 2. Model compounds obtained from Wright Patterson Air Force Research Laboratories for studying hydrolytic and UV stability of PBO chain structure. 




Figure 3. Relevance of model compounds shown in Figure 2 to the PBO repeat unit. 
The a-axis of crystal is radially oriented in a fiber.

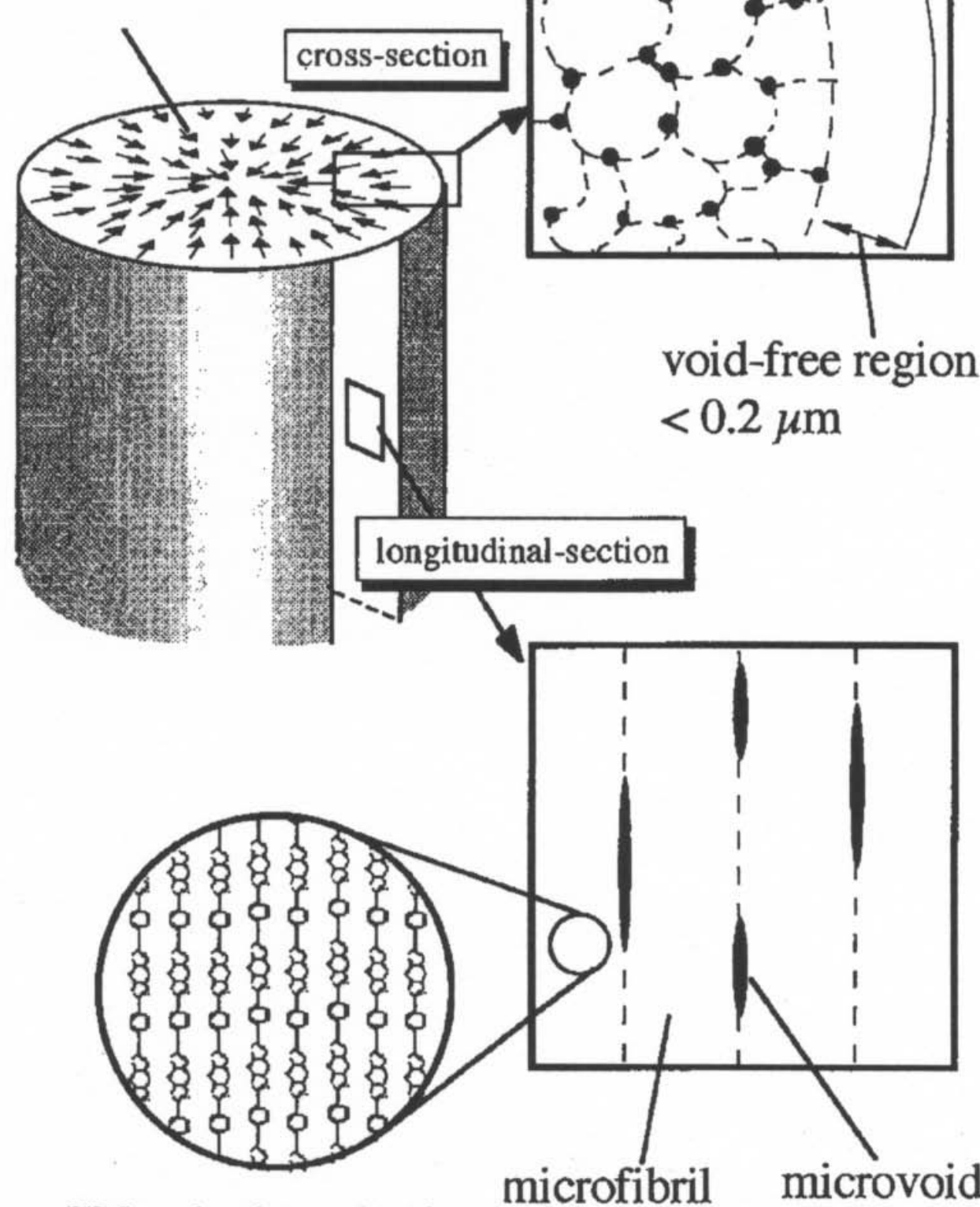

PBO molecules are highly oriented in the microfibril. (orientation factor $>0.95$ )

Figure 4. PBO structure model of AS fiber. (taken from reference 15 with permission) 


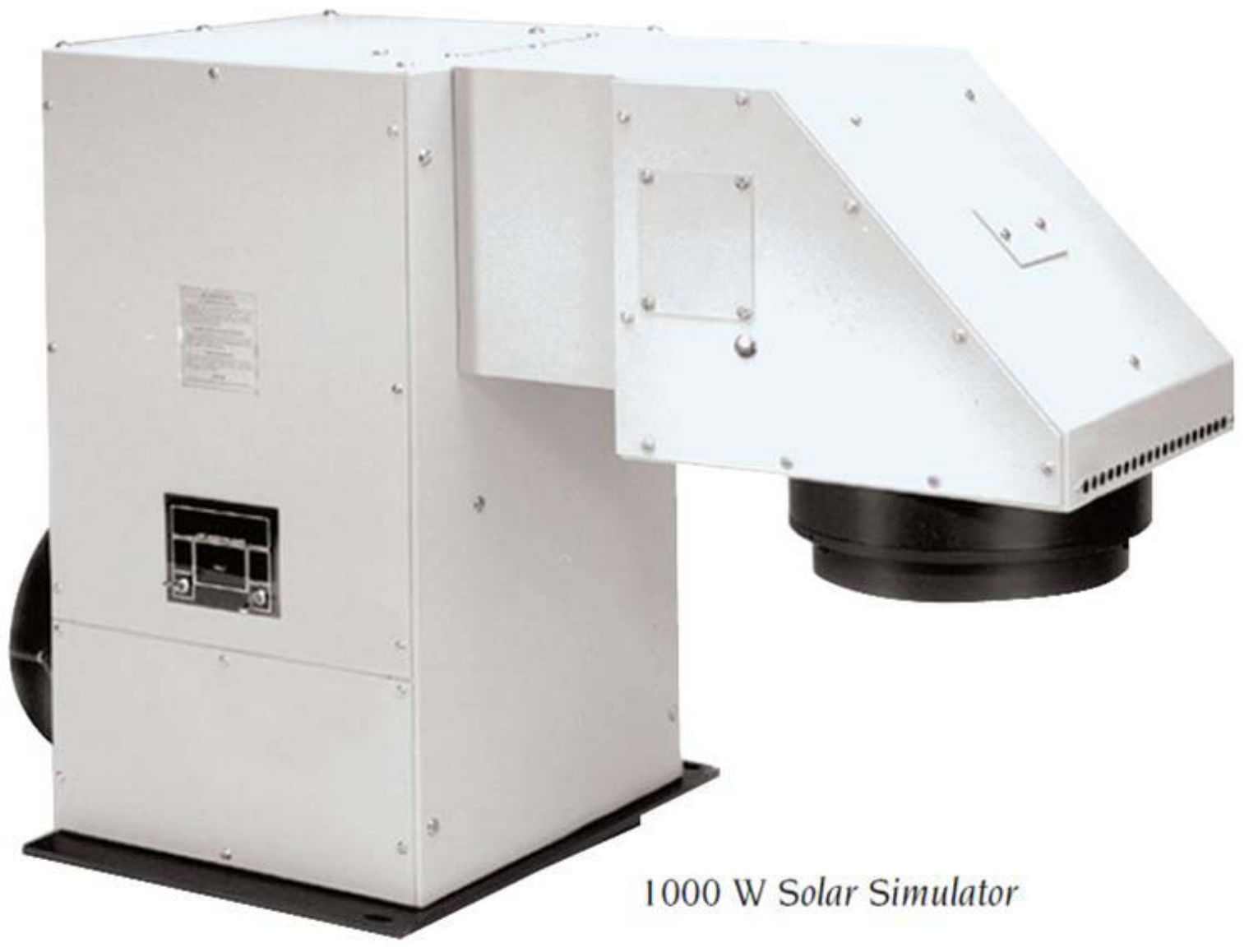

Figure 5. $1000 \mathrm{~W}$ Solar Simulator used for exposing fibers to UV radiation. 


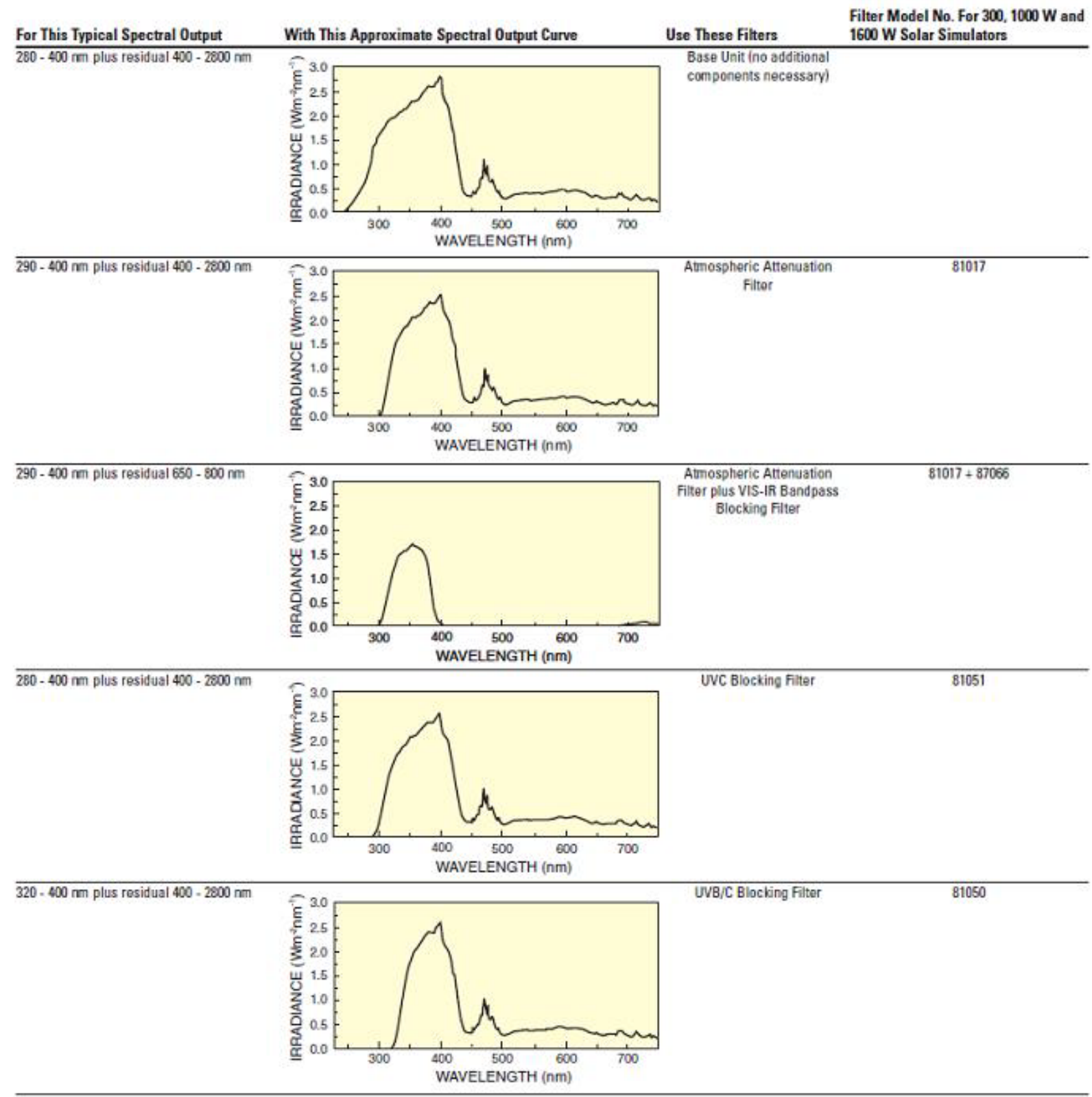

Figure 6. Spectral outputs with selected attachments from $1000 \mathrm{~W}$ UV solar simulator shown in Figure 5. 


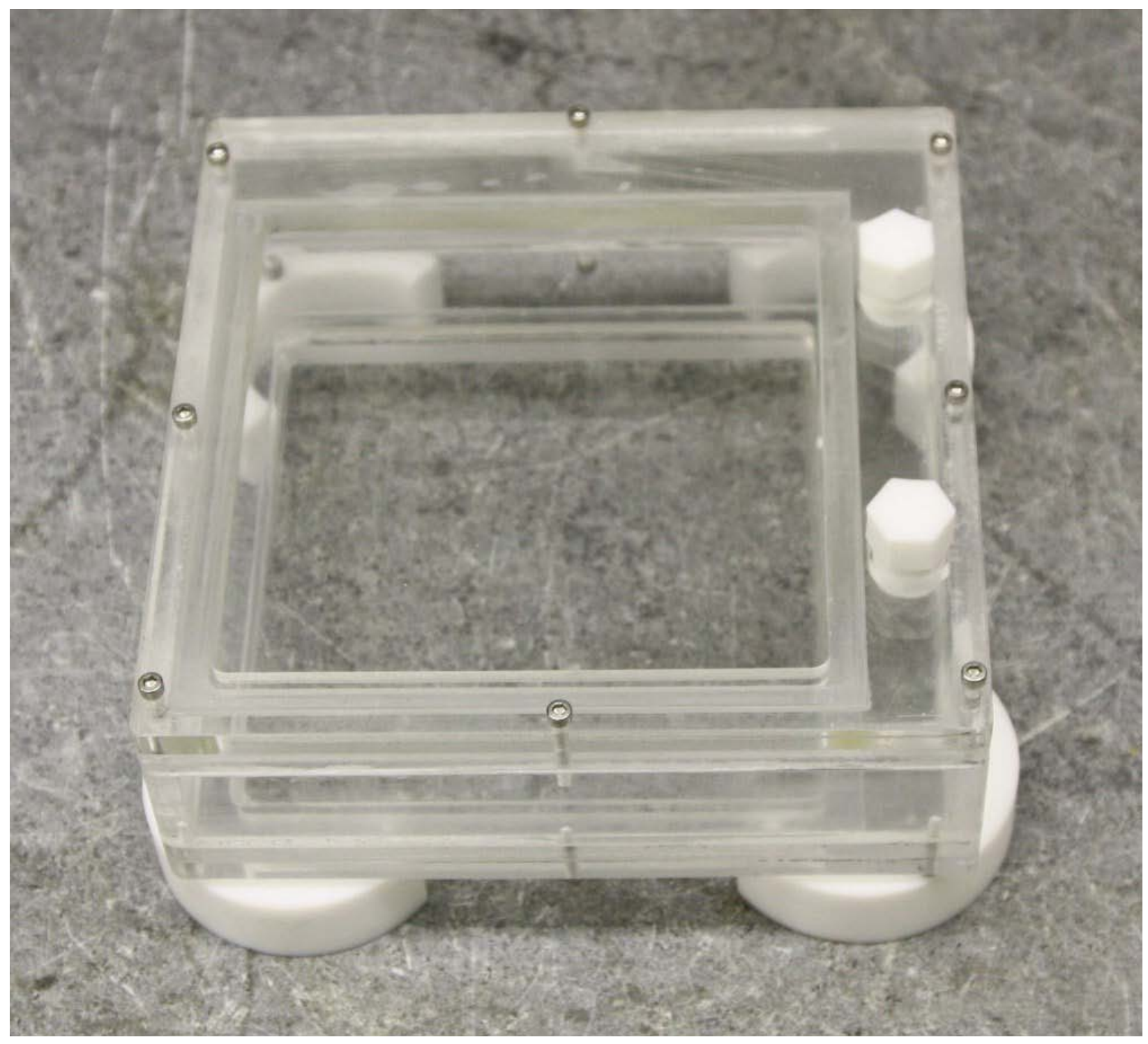

Figure 7. Sealed chamber made of quartz used to exposed acidified PBO fibers to UV radiation using solar simulator shown in Figure 5. 


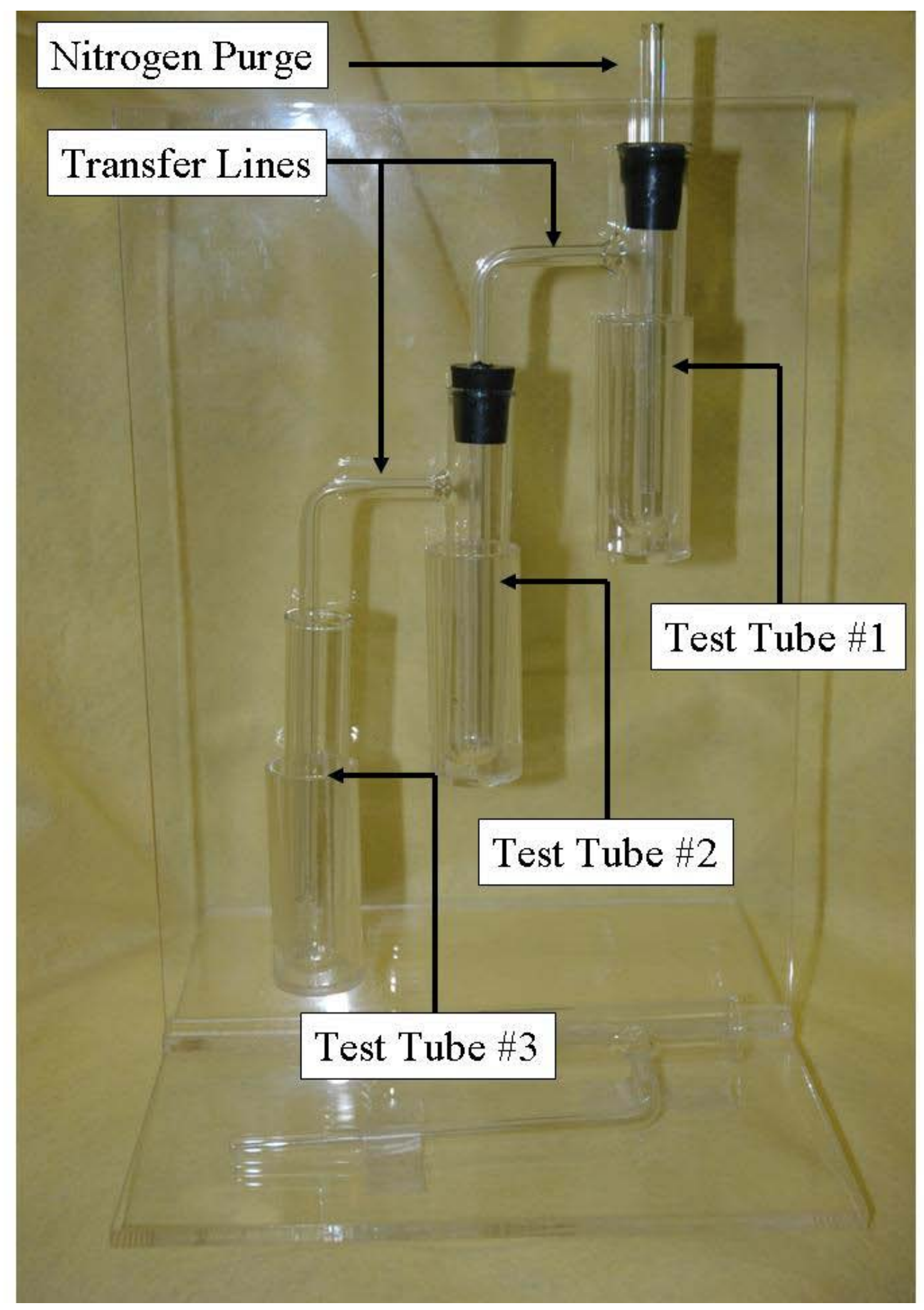

Figure 8. In-Situ Consumption Diazomethane Generator 


\section{Methylation of Carboxylic Acids}<smiles>[R]C(=O)O</smiles>
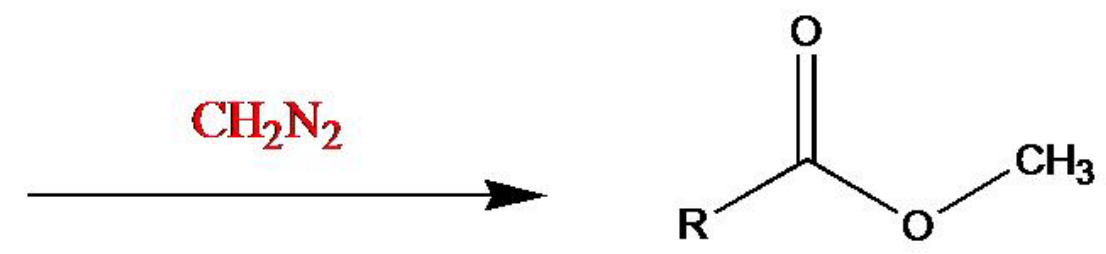

\section{Methylation of Phosphoric Acid}
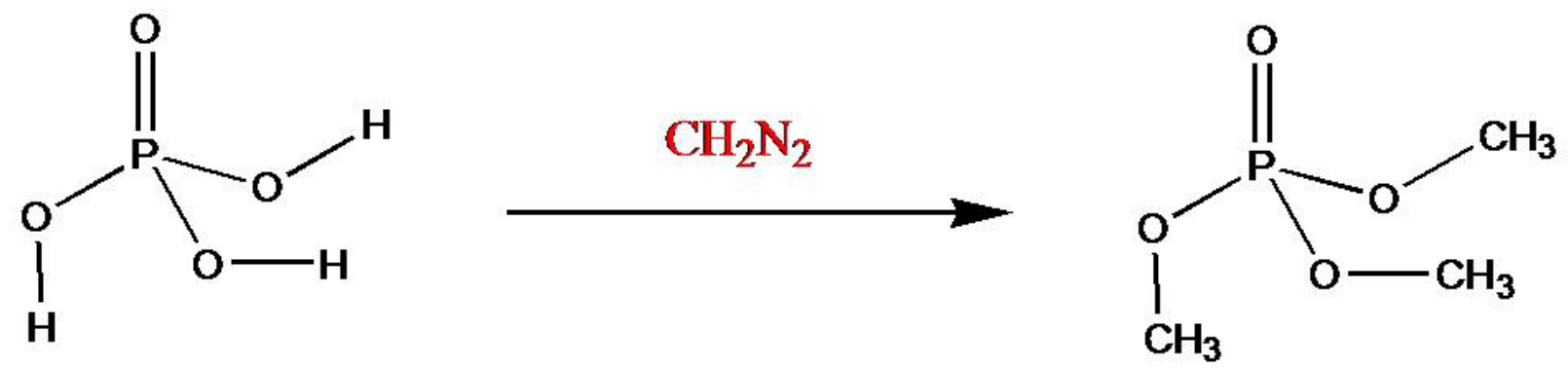

Figure 9. Schematic Representation of carboxylic acid and phosphoric acid methylation by diazomethane. 


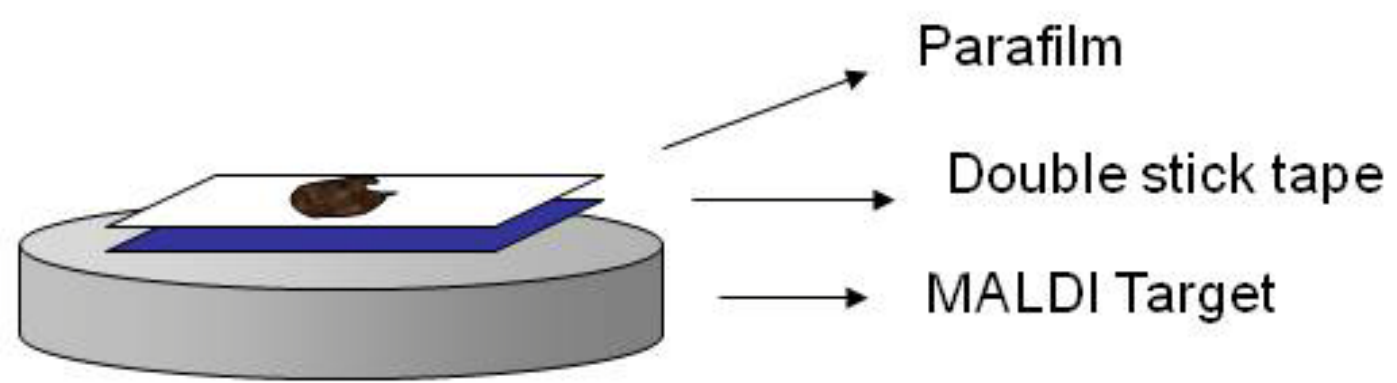

Figure 10. Representation of ground PBO oligomer with small amount of THF/HCl

( $1 \mathrm{ml} / 1$ drop) mixture and pressed with a spatula onto the MALDI target covered by parafilm. 




Figure 11. MALDI-TOF-MS of 2,2'-(1,4-phenylene) bis(benzoxazolyl) (AF1). 


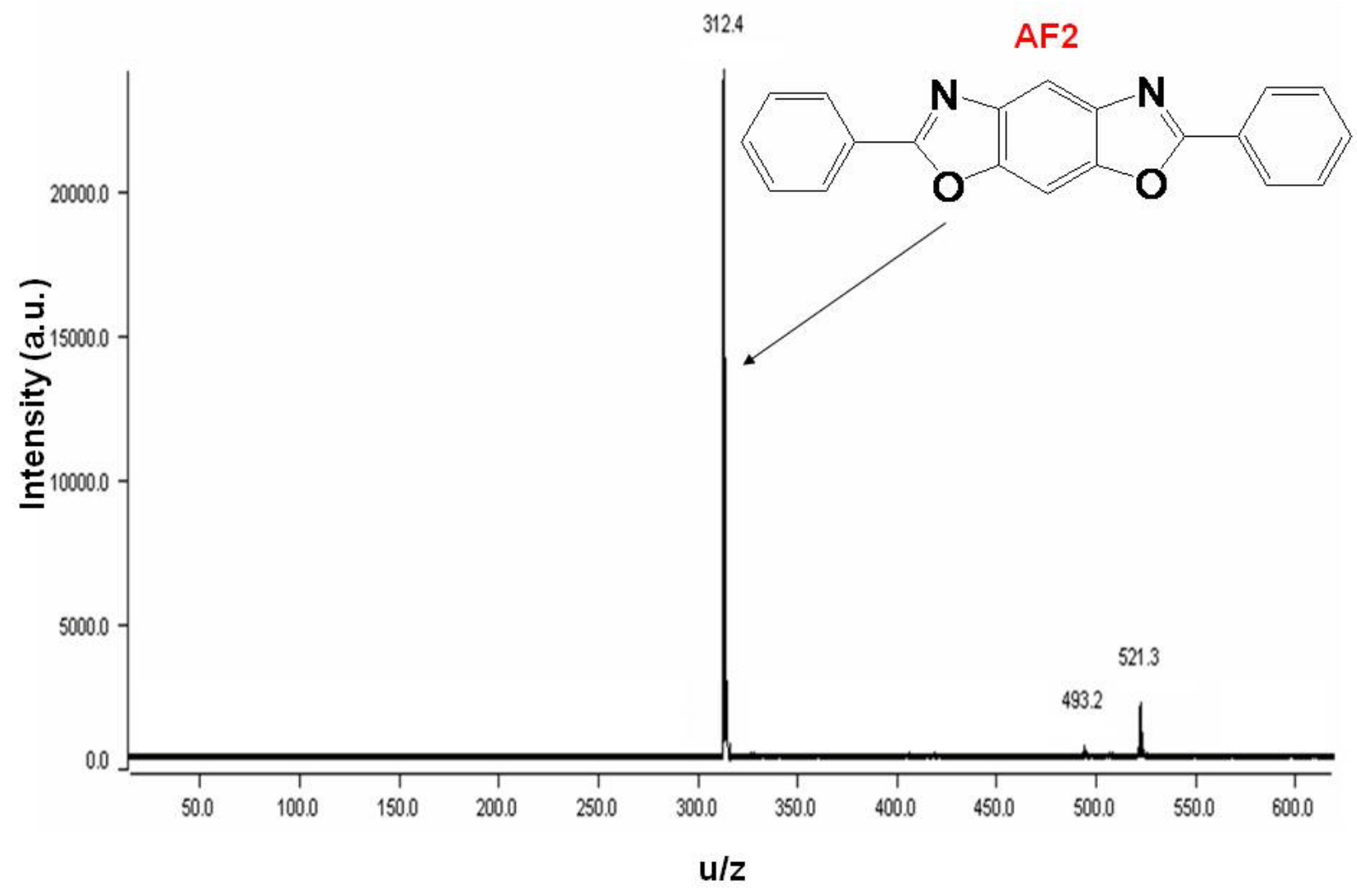

Figure 12. MALDI-TOF-MS of 2,6-diphenylbenzo[1,2-d;5,4-d']bisoxazole (AF2). 




Figure 13. Total ion current trace of methylated phosphoric acid and peaks associated with the methylation procedure scaled in scaled in arbitrary units (a.u.) of intensity. These peaks are identified in Figure 14. 


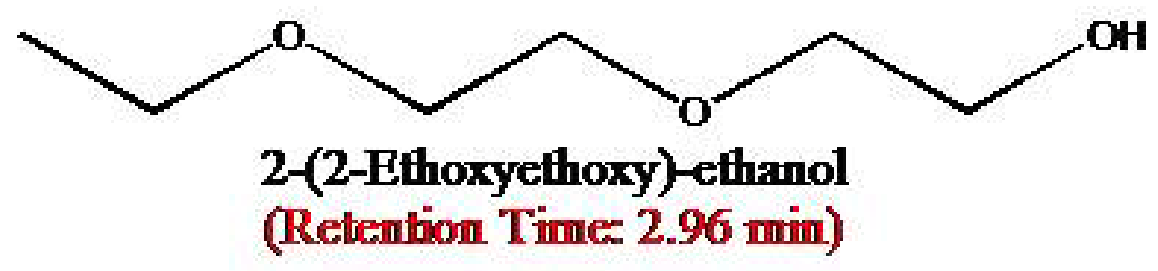<smiles>COS(=O)(=O)c1ccc(C)cc1</smiles>

4-Methyl, methylester Benzenesnlfonic acid

(Retembion. Time $6.32 \mathrm{~min}$ )<smiles>Cc1ccc(S(=O)(=O)N(C)C)cc1</smiles>

N,4-Dimethyl-Benzenessulfonamide

(Retention Time 7.66 min)<smiles>Cc1ccc(S(=O)(=O)N(C)C)cc1</smiles>

N,N,4-Trimethyl-Benzenesnifonamide (Retention Time 9.82 min)

Figure 14. Compounds associated with methylation procedure (see Figure 13). 


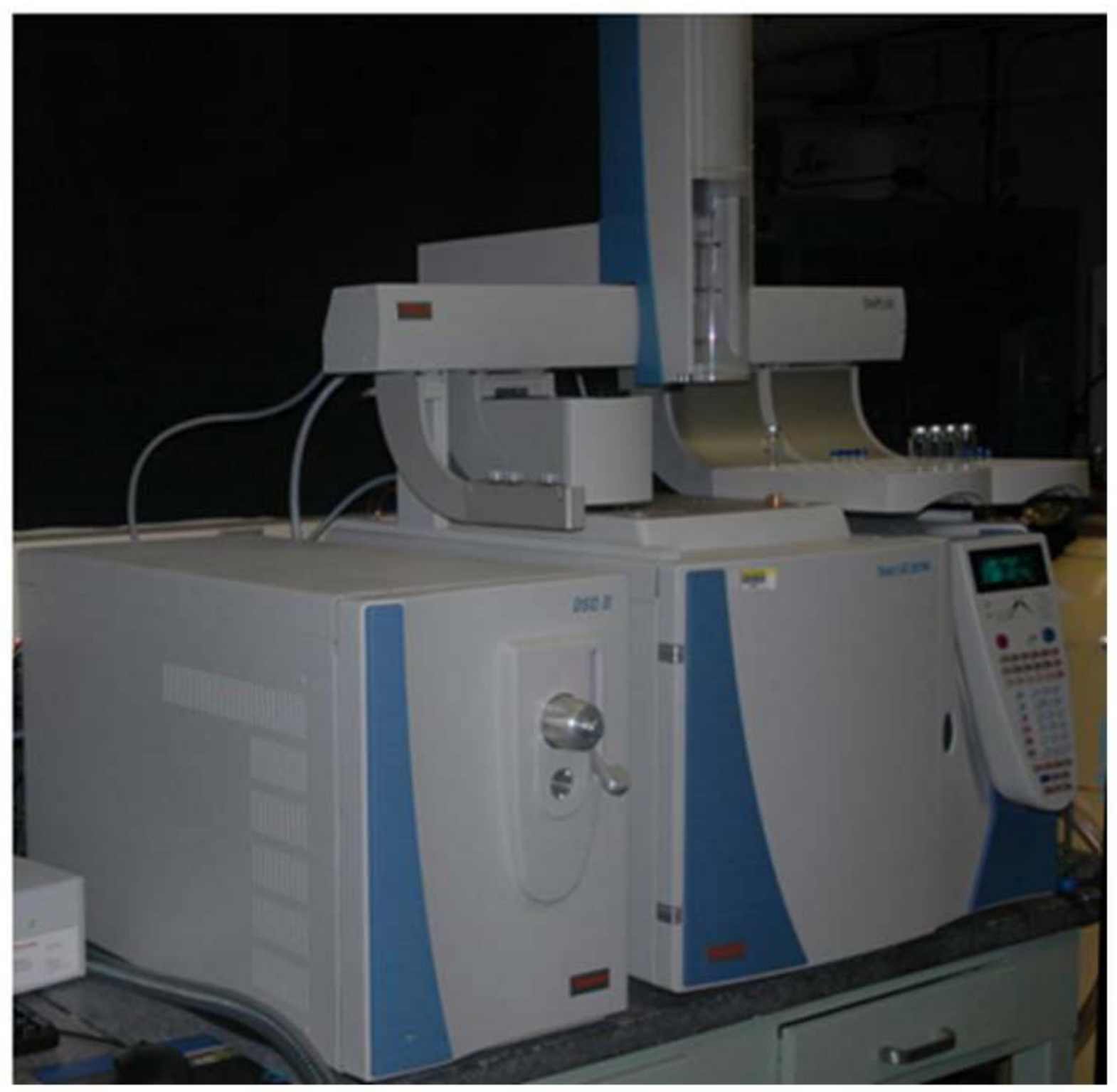

Figure 15. Trace DSQ II mass spectrometer used to identify phosphorus containing species extracted from PBO ballistic fibers. The mass spectrometer was eventually equipped with FPD and NPD detectors to aid in the detection and identification of phosphorus containing species. 
(a)


(c)



(d)

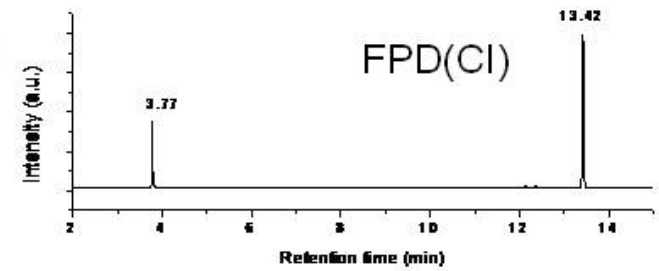

Figure 16. Total ion current (TIC) chromatograms (a) and (c) and parallel flame photometric detector (FPD) chromatograms (b,d) of methylated aqueous extract from PBO fibers tested under electron impact $(\mathrm{EI})(\mathrm{a}, \mathrm{b})$ and chemical ionization $(\mathrm{CI})(\mathrm{c}, \mathrm{d})$ test conditions. A.U. denotes arbitrary units of intensity. 
(a)


(c)



(d)

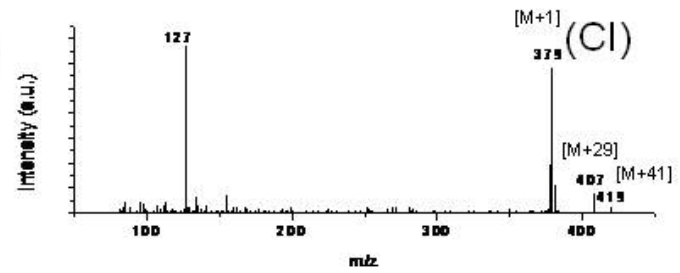

Figure 17. Electron impact (EI) and chemical ionization (CI) spectra of trimethyl phosphate $(a, b)$ and long-chain alkyl phosphate (c,d). A.U. denotes arbitrary units of intensity. 




Figure 1. Mass Spectrum of the TMS Derivative of $1-$ Dodecy $1-\mathrm{d}_{25}$ Phosphate.

Figure 18. Mass Spectrum of the trimethyl silyl derivative of 1-dodecyl- $\mathrm{d}_{25}$ phosphate (adapted from reference 22) showing a very small molecular ion at $420 \mathrm{u}$, indicative to the $\mathrm{C}_{12} \mathrm{D}_{25}$ alkyl group and the fragment ion at $245 \mathrm{u}$ that reflects the subsequent loss of this group as a $\mathrm{C}_{12} \mathrm{D}_{23}$ radical from the molecular ion by the classic McLafferty +1 rearrangement. 




Phosphoric acid attached to PBO oligomer as monoaryl phosphate ester



Triphenyl phosphate

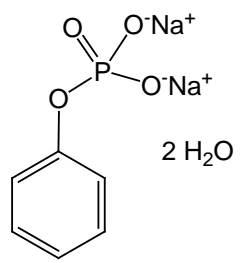

Sodium phenyl phosphate dihydrate dibasic

Figure 19. Chemical structure representation of phosphoric acid chemically bound to PBO oligomer as a monoaryl phosphate ester. To underscore the stability of the monoaryl phosphate ester bond, the chemical structure of triphenyl phosphate (a triaryl phosphate ester) and sodium phenyl phosphate dibasic dihydrate (a monoaryl phosphate ester), which are available commercially, is also shown. 


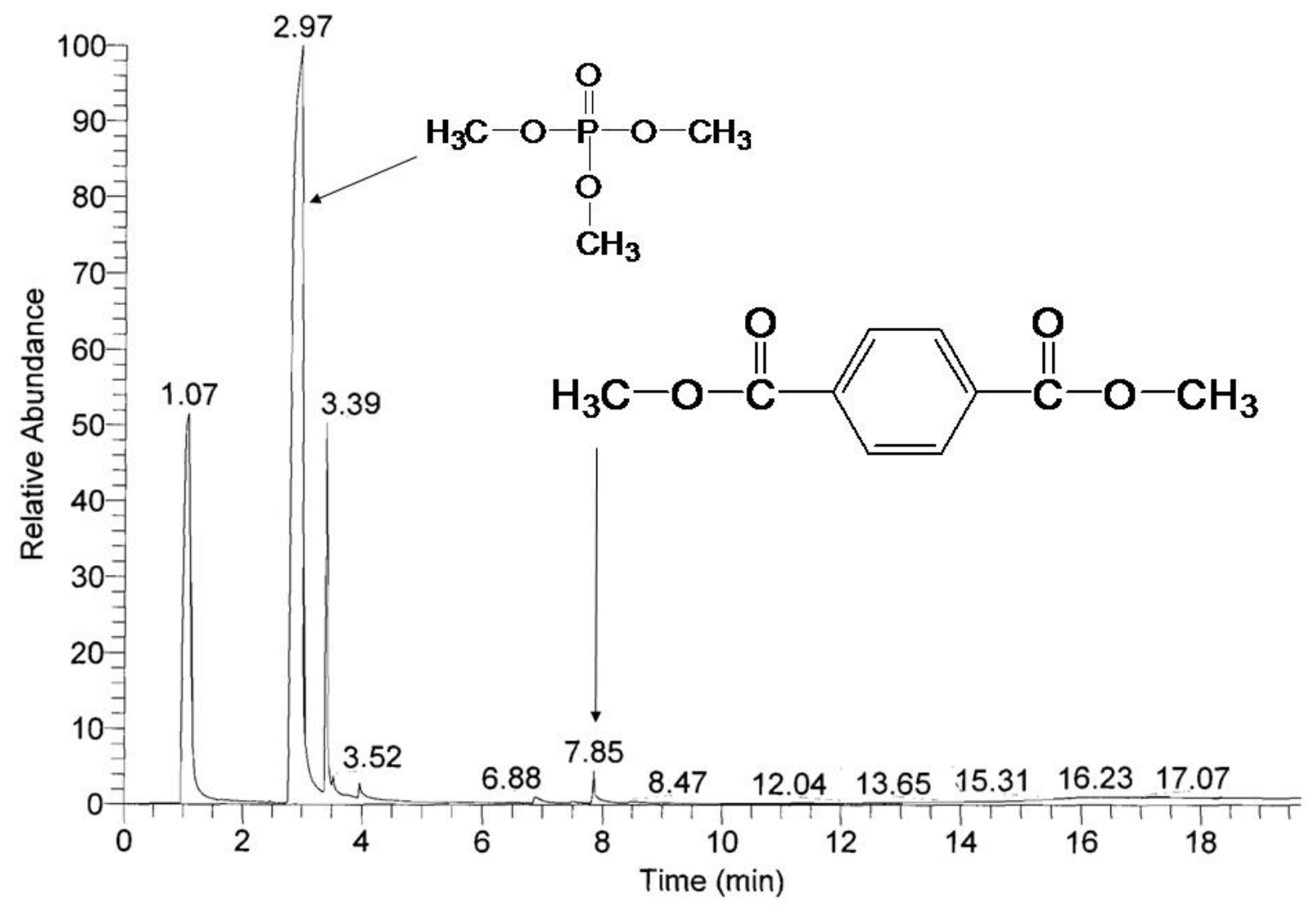

Figure 20. Total ion current trace showing the presence of trimethylphosphate and dimethyl terephthalate extracted from the model PBO oligomers. The unidentified peaks arise from the methylation procedure (see Figure 14). 


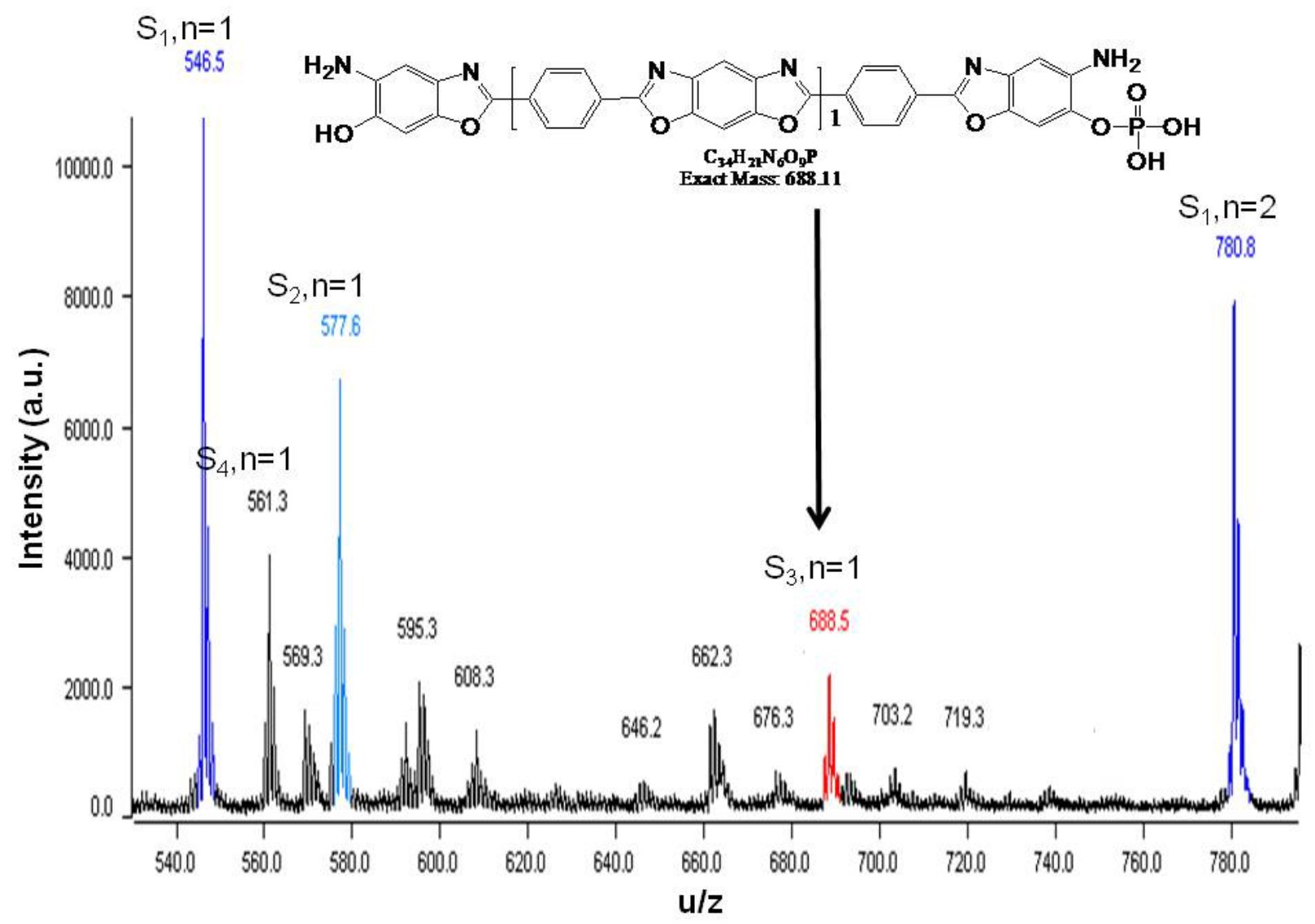

Figure 21. MALDI-TOF MS of low molecular mass model PBO oligomers. A.U. denotes arbitrary units of intensity. 


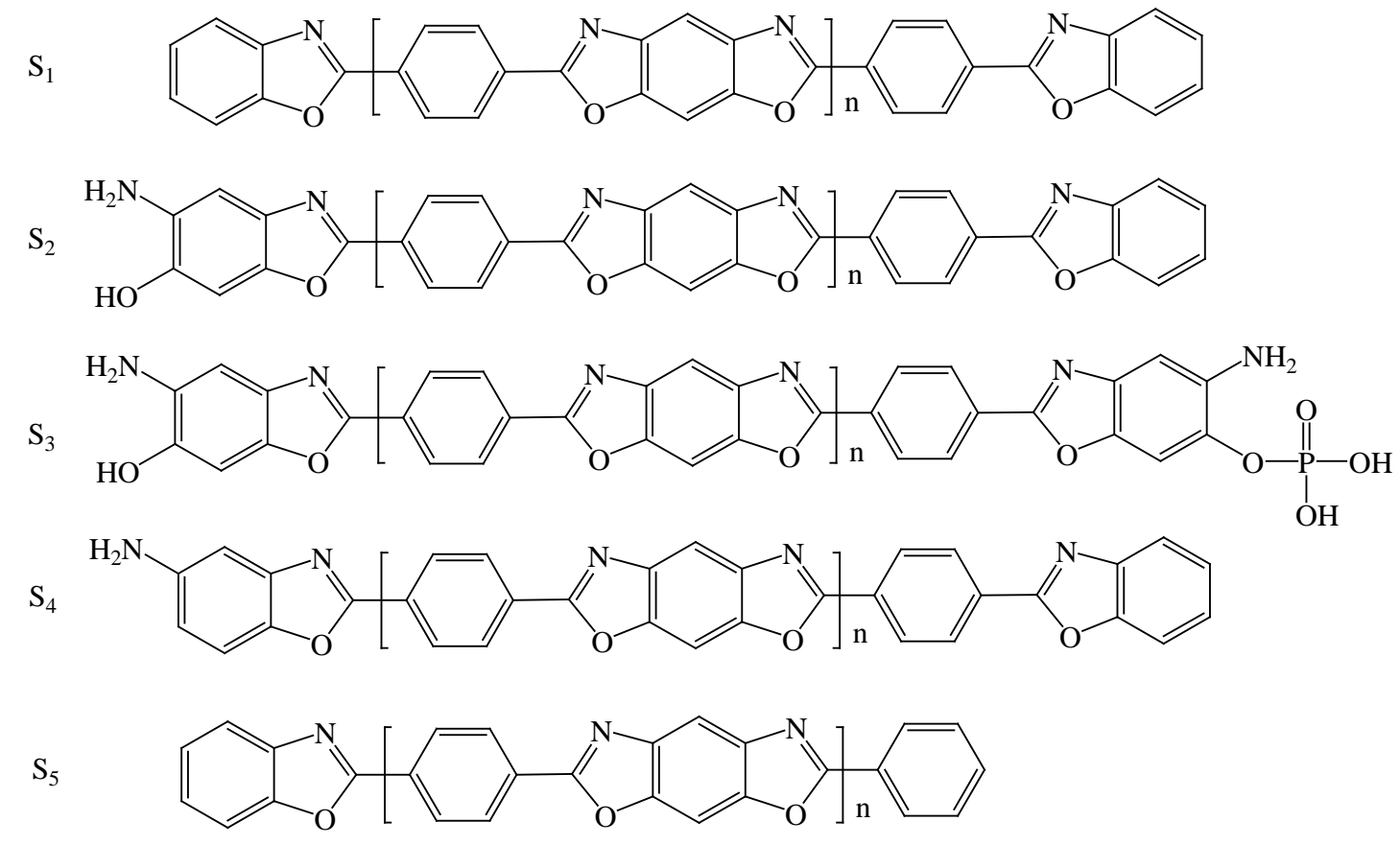

Figure 22. PBO oligomers identified in MALDI mass spectrum shown in Figure 21. 


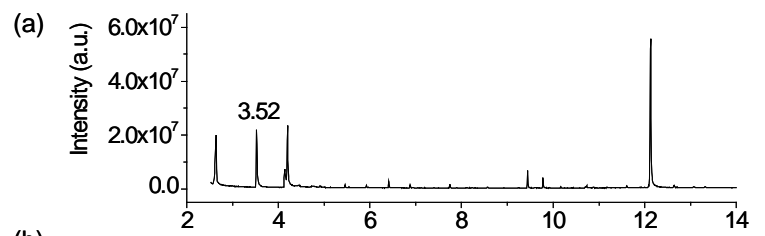

(b)



(c)



(d)

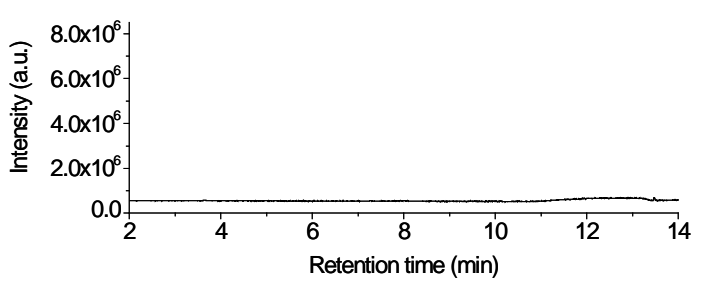

Figure 23. (a) Total ion chromatography (TIC) and (b) FPD spectrum of UV-exposed $2^{\text {nd }}$ Caustic treated $1^{\text {st }}$ PBO fiber Solution in EI mode of GC. (c) TIC and (d) FPD spectrum of UVexposed Water blank in EI mode of GC. A.U. denotes arbitrary units of intensity. 
APPENDIX A 


\section{Standard Operating Procedure for UV-Solar Simulator}

\section{Scope}

This Solar Simulator is designed to simulate various solar conditions. This Solar Simulator produces an unfiltered xenon lamp spectrum. To approximate solar conditions for a variety of solar simulation applications we can modify the spectral output of the arc lamp by mounting various filters to match specific natural solar conditions.

\section{Summary}

Figure 1 shows light pass of a Solar Simulator. We can generate various conditions by changing air mass and/or bandpass filters. It generates uniform, collimated output beams in sizes from $4 * 4$ inches. Our Solar Simulator includes an electronic splitblade shutter. We can externally control this shutter from the Digital Control Panel on the illuminator housing, or via a hand held switch, contact closure, or logic level input. We can do automated exposure control with Digital Exposure Controller.

\section{W Solar Simulators.}
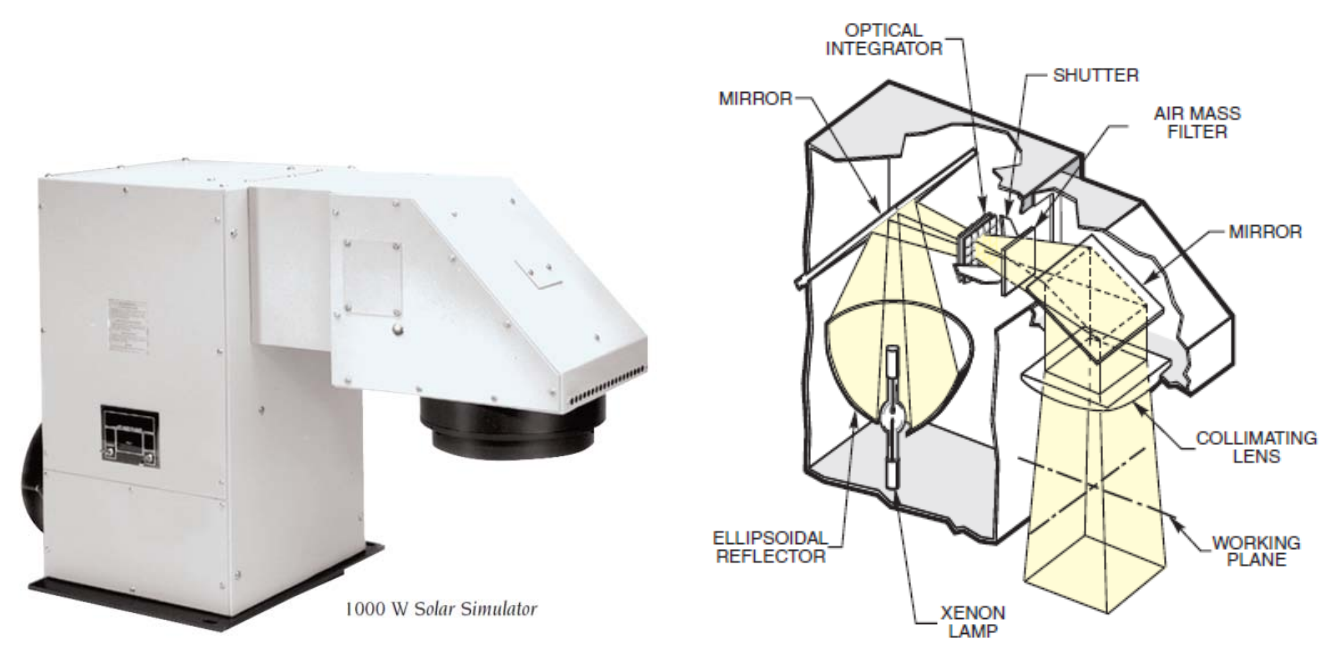

Figure 1. Light of an Oriel Solar Simulator, showing location of air mass filter.

Table1 and figure 2 show the summarized air mass filter type and their function and diagram of air mass filters.

Air Mass 0 Filter (AM 0): this filter corrects the illuminator output to better match the solar spectrum in extraterrestrial space (ie outside the Earth's atmosphere) per ASTM E490-73a. Used alone, this filter generates the Air Mass 0 spectrum. Additionally, it is the first filter element in all other Air Mass filter sets. 
Air Mass 1 Direct Filter (AM 1 Direct): This filter set simulates the solar spectrum at ground level when the sun is directly overhead.

Air Mass 1.5 Direct Filter Set (AM 1.5 Direct): This filter set simulates the the direct solar spectrum with the sun at a zenith angle of $48.2^{\circ}$ (ASTM E891).

Air Mass 2 Direct Filter Set (AM 2 Direct): This filter set simulates the direct solar spectrum with the sun at a zenith angle of 60.10 (ASTM E891).

Air Mass 1.5 Global Filter Set: This filter set corrects the solar simulator output to closely match the total (direct and diffuse) solar spectrum when the sun is at a zenith angle of $48.2^{\circ}$ (ASTM E891).

Table 1. Air Mass Filters

\begin{tabular}{|c|c|c|}
\hline Air Mass Filter Type & Function & Model No(s). \\
\hline$\overline{\mathrm{AM} 0}$ & $\begin{array}{l}\text { Corrects the output of a xenon lamp to } \\
\text { better match the solar spectrum found } \\
\text { outside the earth's atmosphere. }\end{array}$ & 81011 \\
\hline AM 1 Direct & $\begin{array}{l}\text { Simulates the solar spectrum at ground } \\
\text { level when the sun is directly overhead }\end{array}$ & $81011^{*}+81085$ \\
\hline AM 1.5 Direct & $\begin{array}{l}\text { Simulates the direct solar spectrum when } \\
\text { the sun is at a zenith angle of } 48.2^{\circ} \\
\text { (ASTM E891). }\end{array}$ & $81011^{*}+81086$ \\
\hline AM 2 Direct & $\begin{array}{l}\text { Approximates the solar spectrum when } \\
\text { the sun is at a zenith angle of } 60.1^{\circ}\end{array}$ & $81011^{*}+81087$ \\
\hline AM 1.5 Global & $\begin{array}{l}\text { Matches the total (direct and diffuse) } \\
\text { spectrum when the sun is at a zenith } \\
\text { angle of } 48.2^{\circ} \text { (ASTM E892) }\end{array}$ & 81088 \\
\hline
\end{tabular}

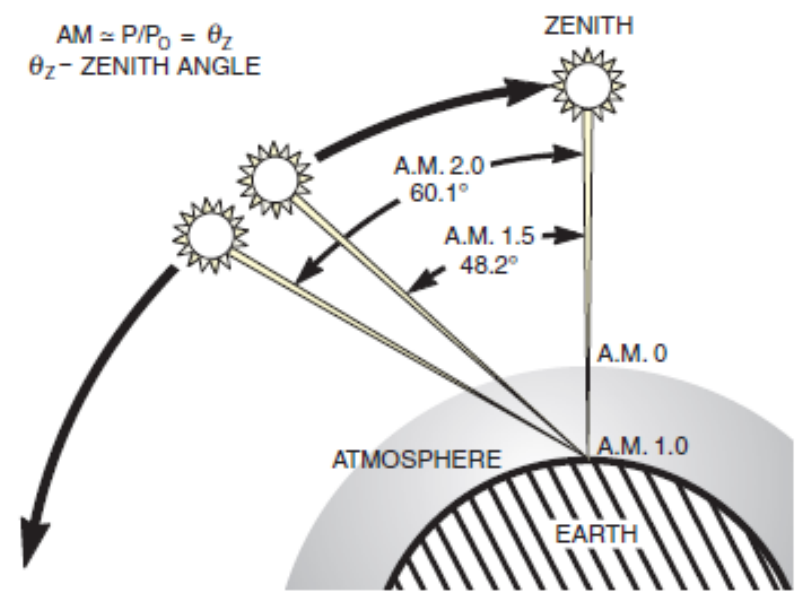

Figure 2. Diagram of solar air masses

Table 2 shows the filters available and spectral output from $230 \mathrm{~nm}$ to $750 \mathrm{~nm}$, when used with this simulator. 
Table 2. Bandpass Filters for Oriel Solar Simulators.






\section{Safety Precautions for chemicals used in this procedure}

Electromagnetic radiation with a wavelength between $10 \mathrm{~nm}$ and $400 \mathrm{~nm}$ is called ultraviolet radiation. Photobiologists use the term UVC for wavelengths to $280 \mathrm{~nm}$, UVB for $280 \mathrm{~nm}$ to $320 \mathrm{~nm}$, and UVA for $320 \mathrm{~nm}$ to $400 \mathrm{~nm}$, although there is disagreement on the $280 \mathrm{~nm}$ boundary. Radiation below $180 \mathrm{~nm}$ is referred to as vacuum UV

\section{UV Radiation from Oriel® Lamp Sources}

The deuterium and arc lamp sources, spectral calibration lamps, Solar Simulators and Flood Exposure Sources produce UV with wavelengths down to $180 \mathrm{~nm}$ and below, depending on the lamp and the envelope material of the lamp (the optics on the lamp housing may block the shorter UV wavelengths). Our Quartz Tungsten Halogen Sources emit some radiation down to $220 \mathrm{~nm}$. The UV intensity levels from some of these sources may be higher than those from the sun, and shorter wavelengths may be present. Carefully read this discussion as well as the Safety Instructions that are included with every UV source we ship, before operating the source.

\section{Exposure Hazards}

Exposure to UV radiation, even for short periods of time, can be hazardous. The damage depends upon the exposure time, the intensity of the radiation, the wavelength, and the individual's sensitivity to UV. Since we cannot sense (see) UV radiation, we are not protected by any aversion or blink response.

\section{The Skin}

UVB and UVC cause sunburn (erythema) and pigmentation (tanning). Long-term exposure results in loss of skin elasticity (premature aging). There is a well established connection between wavelengths below $320 \mathrm{~nm}$ and skin cancer (basal cell and squamous cell carcinoma) and growing concern over possible longer wavelength involvement. Action spectra for carcinogenesis peak at $\sim 290 \mathrm{~nm}$.

\section{The Eye}

Corneal absorption of UVB and UVC may cause conjunctivitis and a corneal inflammation called photo keratitis. Initial symptoms of photo keratitis are: slight discomfort, which, after prolonged exposure, can develop into a temporary yet severe discomfort, an inability to look at bright light, and the loss of clear vision. Conjunctivitis (welder's eye) produces an uncomfortable sensation, similar to sand in the eye. Our innocuous pencil style mercury calibration lamps can cause this problem.

Prolonged exposure to longer ultraviolet radiation, particularly UVA, may cause cataracts to form in the eye lens. Longer wave UVA may also penetrate to the retina and result in "blue blindness". 


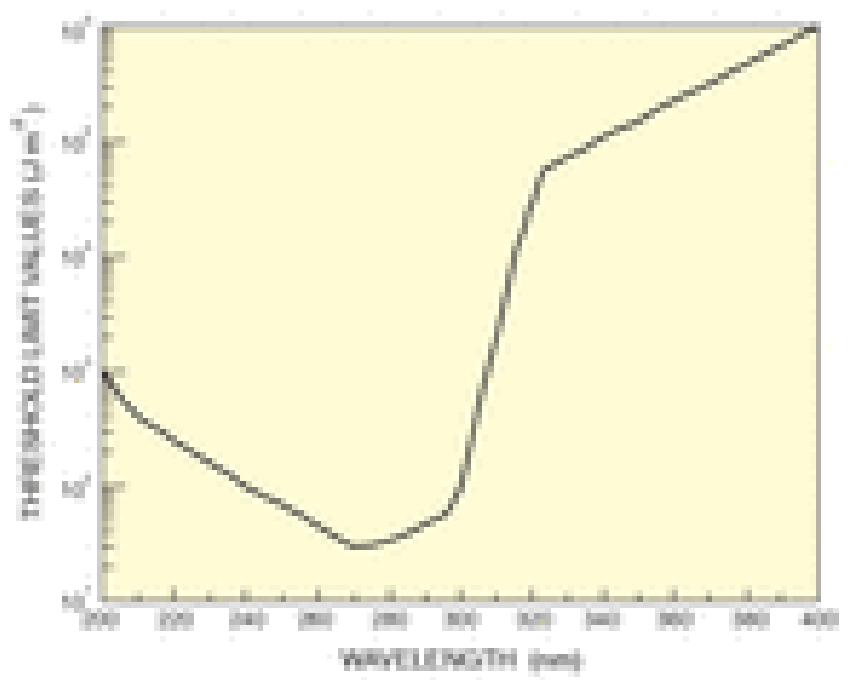

Fig. 2 Threshold Limit Values for occupational exposure to ultraviolet radiation in an 8 hour period. These values for exposure of the eye or the skin apply to UV radiation from arcs, gas and vapor discharges, fluorescent and incandescent sources, and solar radiation, but they do not apply to UV lasers. (ACGIH ISBN: 0-9367-12-99-6)

\section{UV Safety Precautions}

The simplest thing to do if you don't need the ultraviolet is to get rid of it at the source. Use a lens or filter to accomplish this. If you require the UV then there are several precautions you should take to minimize exposure and reduce the hazards.

- Limit access to areas where UV sources are used

- Post warning signs at the entrance to labs or other work areas using UV sources

- Wear protective eyewear and gloves

- Cover arms and neck and limit exposure time

- Never look directly at the beam

- Use a manual or electronic shutter to close the beam when the source is not in use

- Use enclosed beam paths where possible

\section{Ozone}

If you note ozone odor smell persistently in an area remote from the fans, check the ozone levels and improve ventilation of the area.

\section{Lamp Hazards}

There is always a danger of lamp explosion due to mechanical failure

- Never touch the lamp with bare fingers or other contaminates

- Always wear appropriate gloves and impact-resistant goggles when handling the lamp

- Avoid any mechanical strain during handling

- Do not operate the lamp without all housing panels in place 


\section{Safety Guidelines}

The U.S. National Institute for Occupational Safety and Health (NIOSH) recommends that exposure to UV energy be controlled and limited in the work place. Recommended maximum exposures are available, but these do not apply to photosensitive individuals or those exposed to photosensitizers.

The total intensity from 320 to $400 \mathrm{~nm}$ hitting an unprotected eye should not exceed $10 \mathrm{Wm}^{-2}$ for periods longer than 1000 seconds. For shorter exposure times, the energy density should not exceed $104 \mathrm{Jm}^{-2}$

\section{Procedure}

\section{Quick Start UV Solar simulator}

1. Turn on the lamp power supply

(Do this process if you changed light source, usually this set up has done. $\rightarrow$ Set it to the maximum power level that you will allow your lamp to operate at, near the end of its life: normally $10 \%$ higher then desired operating point. (e.g. $1100 \mathrm{~W}$ for a nominally $1000 \mathrm{~W}$ lamp - check for the safe power levels for the type of lamp you are using))

2. Start Lamp

3. Allow the system to stabilize for approximately 15 minutes.

4. Turn on Digital Exposure Controller while holding down the STOP/CLOSE button. (This action makes sure that you are starting at factor default set of values.)

5. Using TIMER/FLUX and SELECT buttons scroll until the $\mu$ A LED is lit.

6. Check that light sensor is reading somewhere between 1 and $500 \mu \mathrm{A}$ for best performance (use the START button to open the shutter, if present). ( $\rightarrow$ Modify your light coupling method if the reading is outside of this range.)

7. Press the AUTO SETUP button and hold it until the AUTO-SETUP LED starts flashing (this may take up to $5 \mathrm{~s}$ ).

$(\rightarrow$ The unit will bow open the shutter, if present, and investigate lamp's output changes in response to power supply output changes. This data is used to optimize the intensity control function. $\rightarrow$ At the end of this step, the Digital Exposure Controller will save the min-max values of the possible control range and use its mid-point for a starting reference level. This will typically result, when FLUX CONTROL is later turned on, in power supply setting. $\rightarrow$ The shutter will now close and AUTO-SETUP LED will go off. Typically 10 to 20 seconds elapse from the beginning to the end of this function. $\rightarrow$ If FAULT LED lights up, see recovery section on the following page.

8. If closing of the shutter makes the $\mu \mathrm{A}$ readings go close to zero $\mu \mathrm{A}$ 's (light sensor mounted after the shutter), IDLE mode should be selected to keep power supply under control in this situation.

$(\rightarrow$ Use the SET/ENTER and SELECT buttons to scroll through the LED's until IDLE LED is lit. $\rightarrow$ Press SET/ENTER button for a moment until the IDLE LED flashes. $\rightarrow$ Use UP/DOWN buttons to choose 1 from the 0 and 1 options for the IDLE function. $\rightarrow$ Press SET/ENTER to save this selection.)

9. Press the FLUX CONTROL button to enable stable exposure.

10. Set exposure mode to automatic. 
$(\rightarrow$ Light up the MODE LED (TIMER/FLUX and SELECT buttons). $\rightarrow$ Enter the mode selection (SET/ENTER) and scroll to AUTO-TIME LED (UP/DOWN buttons). $\rightarrow$ Save the selection (SET/ENTER).)

11. Light up the OPEN TIME LED (TIMER/FLUX and SELECT buttons) and enter the length of desired exposure (SET/ENTER, UP/DOWN, SELECT, SET ENTER buttons).

12. Press the START button to run your exposure.

\section{Recovery from Fault Condition}

1. Turn both the light source power supply and the Digital Exposure Controller off.

2. Disconnect the Power Supply Output control cable from the Digital Exposure Controller

3. Turn on the power supply and power up the lamp to the maximum setting

4. Turn on the Digital Exposure Controller.

5. Connect the Power Supply Output control cable back to the controller.

6. This resets the Power Supply operating point by releasing control from the Digital Exposure Controller, but it does nothing to resolve the cause of the fault. Please modify whatever condition caused the fault before running the auto setup again ( $\rightarrow$ Check power supply limit settings $\rightarrow$ Check light sensor signal level $\rightarrow$ Check any other potentially “offending” candidates in your particular system) 
APPENDIX B 


\section{Standard Operating Procedure for Derivatization with Diazomethane}

\section{Scope}

This procedure is designed to alkylate residual acid impurities that may be found on poly $(p$ phenylenebenzoxazole) [PBO] fibers and derivatize nanoscale defect sites along the polymer chain of PBO fibers and PBO model compounds. This methodology is needed so that these analytes can be analyzed by gas chromatography (GC) / mass spectrometry (MS), GC / Fourier transform infrared (FTIR) spectrometry, and matrix assisted laser desorption ionization (MALDI) - time of flight (TOF) MS. In addition, this procedure will be used to methylate any residual phosphoric acid $\left(\mathrm{H}_{3} \mathrm{PO}_{4}\right)$ that may be extracted from PBO fibers by soxhlet extraction procedures.

\section{Summary}

Diazomethane is highly toxic, with early synthetic procedures stressing extreme care be exercised in the preparation and use of this material [1]. In this procedure, diazomethane is generated from the action of alkali on N-methyl-N-nitroso- $p$-toluenesulfonamide (diazald, see Figure 1) that has been shown to be a safe precursor for generating diazomethane.<smiles>Cc1ccc(S(=O)(=O)N(C)N=O)cc1</smiles>

N-methyl-N-nitroso-p-toluenesulfonamide

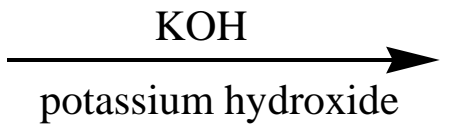

(Diazald (diazomethane precursor))

Figure 1. Reaction of potassium hydroxide on N-methyl-N-nitroso-p-toluenesulfonamide

Nitrogen gas passed through test tube \#1 and into test tube \#2 where the diazomethane is generated. The diazomethane and nitrogen gas mixture is then bubbled into a collecting vial (test tube \#3) that contains methyl-t-butyl ether and the material to be derivatized (see Figure 2). Material need not be soluble in methyl-t-butyl ether 


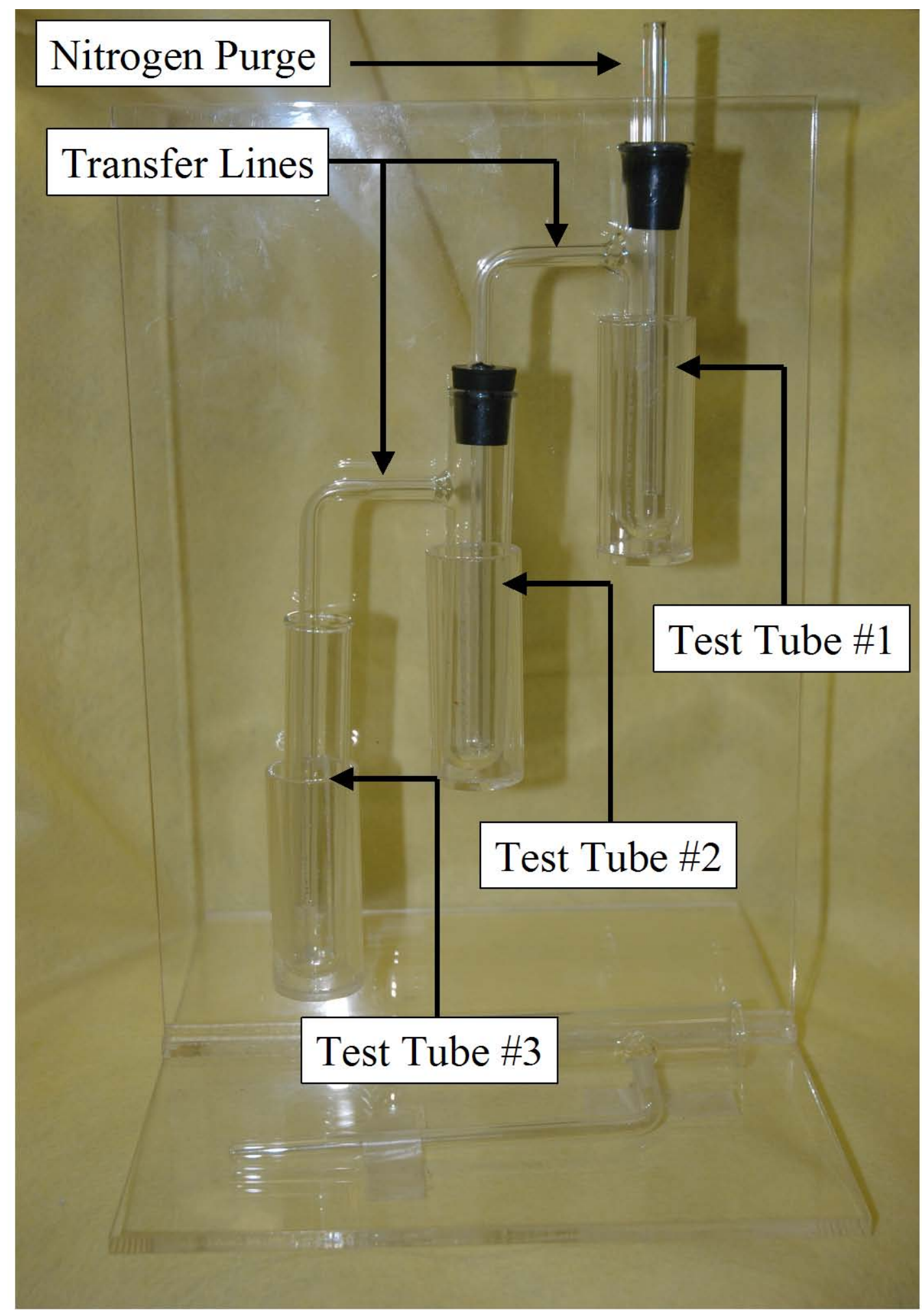

Figure 2. In-Situ Consumption Diazomethane Generator 
The procedure described herein differs from the methods described in references 2 and 3 by the in-situ consumption of the millimole quantities of diazomethane that are generated from the $\mathrm{N}-$ methyl-N-nitroso- $p$-toluenesulfonamide. This approach further minimizes the explosive hazards associated with this reaction. Diazomethane is commonly used to derivatize carboxylic acids (4) and chlorinated herbicides (2), phenols, and enols (5). Consistent with these results, diazomethane is also known to derivatize phosphoric acid to trimethylphosphate (6), a potential residual acid impurity in PBO fibers (see Figure 3).

Methylation of Carboxylic Acids



Methylation of Phosphoric Acid


Figure 3. Schematic Representation of carboxylic acid and phosphoric acid methylation by diazomethane.

In addition, it is known that diazomethane reacts with amides, amines, hydroxyl groups on benzene rings, and other compounds containing labile hydrogen atoms. Interestingly, the commonly accepted hydrolytic and UV degradation pathways for PBO, suggests the possible formation of degradation products with labile hydrogen atoms (7). Since PBO does not contain labile hydrogen atoms, the diazomethane procedure may be useful in illuminating the presence of degradation sites along the chain of PBO fibers.

\section{Safety Precautions for chemicals used in this procedure}

Diazomethane is a toxic carcinogen that can explode under certain conditions. The following general precautions must be followed when using this material:

1) Use only a well-ventilated hood - do not breathe vapors.

2) Use a safety screen.

3) Use mechanical pipetting aides

4) Do not heat above $90{ }^{\circ} \mathrm{C}$ - EXPLOSION may result.

5) Avoid grinding surfaces, ground glass joints, sleeve bearings, glass stirrers EXPLOSION may result. Therefore, all glassware used in this reaction must be fire-polished. No broken or chipped glass can be used with this reaction.

6) Store away from alkali metals - EXPLOSION may result. 
7) Solutions of diazomethane decompose rapidly in the presence of solid materials such as copper powder, calcium chloride, and boiling chips.

8) The diazomethane generation apparatus used in esterification procedure produces micromolar amounts of diazomethane to minimize safety hazards.

\section{phosphoric acid \\ C Corrosive}

Risk Statements 34

34: Causes burns

Safety Statements 26-36/37/39-45

26: In case of contact with eyes, rinse immediately with plenty of water and seek medical advice 36/37/39: Wear suitable protective clothing, gloves and eye/face protection

45: In case of accident or if you feel unwell, seek medical advice immediately (show label where possible)

$\operatorname{diazald}^{\circledR}(1)$

E Explosive

Xi Irritant

Risk Statements 2-36/37/38-43

2 Risk of explosion by shock, friction, fire or other sources of ignition 36/37/38: Irritating to eyes, respiratory system and skin

43: May cause sensitization by skin contact

Safety Statements $\underline{15-26-35-36 / 37}$

15: Keep away from heat

26: In case of contact with eyes, rinse immediately with plenty of water and seek medical advice 35: This material and its container must be disposed of in a safe way

36/37: Wear suitable protective clothing and gloves

potassium hydroxide

Hazard Codes $\underline{C}$

C Corrosive

Risk Statements 22-35

22: Harmful if swallowed

35: This material and its container must be disposed of in a safe way

Safety Statements 26-36/37/39-45

26: In case of contact with eyes, rinse immediately with plenty of water and seek medical advice 36/37/39: Wear suitable protective clothing, gloves and eye/face protection

45: In case of accident or if you feel unwell, seek medical advice immediately (show label where possible)

methyl-t-butyl ether

Hazard Codes F, Xi

F Highly Flammable

Xi Irritant 
Risk Statements $\underline{11-38}$

11: Highly Flammable

38: Irritating to the skin

Safety Statements $\underline{9-16-24}$

9: Keep container in a well-ventilated place

16: Keep away from sources of ignition - No smoking

24: Avoid contact with the skin

trimethylphosphate

Hazard Codes $\underline{T}$

T Toxic

Risk Statements $\underline{46-22-40}$

46: May cause heritable genetic damage

22: Harmful if swallowed

40: Possible risk of irreversible effects

Safety Statements $\underline{53-36 / 37-45}$

53: Avoid exposure - obtain special instruction before use

36/37: Wear suitable protective clothing and gloves

45: In case of accident or if you feel unwell, seek medical advice immediately (show label where possible)

carboxylic acid

none

methanol

Hazard Codes F, T

F Highly Flammable

T Toxic

Risk Statements $\underline{11-23 / 24 / 25-39 / 23 / 24 / 25}$

11:_Highly Flammable

23/24/25: Toxic by inhalation, in contact with skin and if swallowed

39/23/24/25: Toxic: danger of very serious irreversible effects through inhalation, in contact with skin and if swallowed

Safety Statements 7-16-36/37-45

7: Keep container tightly closed

16: Keep away from sources of ignition - No smoking

36/37: Wear suitable protective clothing and gloves

45: In case of accident or if you feel unwell, seek medical advice immediately (show label where possible)

Diethyl ether

Hazard Codes $\mathrm{F}+, \mathrm{Xn}$

F+: Extremely Flammable 
Xn: Harmful

\section{Risk Statements $\underline{12-19-22}$}

12: Extremely Flammable

19: May form explosive peroxides

22: Harmful if swallowed

Safety Statements $\underline{\text { 9-16-29-33 }}$

9: Keep container in a well-ventilated place

16: Keep away from sources of ignition - No smoking

29: Do not empty into drains

33: Take precautionary measures against static discharges

\section{Glassware}

A. $\quad 2$ fire-polished test tubes that have glass transfer lines for the nitrogen $\left(\mathrm{N}_{2}\right)$ gas to pass to the adjacent test tube (see Figure 1).

B. 1 fire-polished test tube with no transfer line.

C. 1 fire-polished $\mathrm{N}_{2}$ purge inlet tube.

D. All test tubes must have openings for rubber stoppers.

E. 2 rubber stoppers that contain a single hole. 1 rubber stopper will be fitted with the $\mathrm{N}_{2}$ purge inlet tube. The second rubber stopper will be fitted on the transfer line of test tube \#1 at a height that will allow it to fit into test tube \#2.

F. Plexiglass holder that holds the three test tubes and acts as an initial safety shield.

\section{Reagents}
A. Carbitol (Di(ethylene glycol) ethyl ether), Aldrich, 537616100 g bottle (keep refrigerated). 90 day check for peroxides
B. Diazald (N-methyl-N-nitroso-p-toluenesulfonamide), Aldrich D2,800-0, 100 g bottle ( keep refrigerated).
C. Potassium Hydroxide pellets, $\mathrm{KOH}$, ACS grade.
D. Ethyl Ether, Nanograde un-preserved, peroxide-free (stored under hood). Single use only. Test for peroxides prior to use.
E. $\quad 37 \% \mathrm{KOH}$ : Weigh 37 grams of $\mathrm{KOH}$ into a $100 \mathrm{ml}$ volumetric flask and bring to volume with nanograde water (store at $4{ }^{\circ} \mathrm{C}$ ).
F. Methanol, Chromatographic Grade

\section{Procedure}

A. Diazomethane Preparation: Refer to Figure 1.

1. Place diazomethane generator shown in Figure 1 into a clean hood that contains no heat or spark sources.

2. Use a $\mathrm{N}_{2}$ cylinder and install cylinder according to safety guidelines. 
3. Add a sufficient amount of ethyl ether to test tube \#1 so that when the purge inlet tube is in place the volume content of the ether is at least $50 \%$ in the test tube volume but lower than the glass transfer arm that goes to test tube \#2.

4. Place the stopper with connecting tubing on tube $\# 1$ and adjust nitrogen purge so that liquid ethyl ether does not bubble out of the transfer arm into test tube \#2.

5. Turn off $\mathrm{N}_{2}$ purge.

6. Add 2-4 ml of carbitol to test tube \#2.

7. Add $4 \mathrm{ml}$ of $37 \% \mathrm{KOH}$ to test tube \#2.

8. Place test tube \#1 into test tube \#2 via the rubber stopper making sure that the glass transfer arm from test tube \#1 is well below the liquid level in test tube \#2. The liquid level in test tube \#2 with the glass transfer arm from test tube \#1 should be approximately $\underline{50 \%}$ of the volume of the test tube. If needed add more carbitol.

9. Turn on the $\mathbf{N}_{2}$ purge and make sure that the liquids in test tubes \#1 and \#2 remain in their respective chambers.

10. Turn off $\mathrm{N}_{2}$ purge.

11. Place sample to be methylated into test tube \#3 with (2 to 4) ml of methanol.

12. Connect test tubes 1,2 , and 3 together. Start $\mathrm{N}_{2}$ purge and make sure that the $\mathrm{N}_{2}$ bubbles into test tube $\# 3$ below the liquid line and at an acceptable rate. If needed add more methanol to tube \#3.

13. With the $\mathrm{N}_{2}$ purge still on, gently break the rubber seal between test tube \#1 and test tube \#2.

14. Add (0.2 to 0.4$) \mathrm{g}$ (solid) of the diazald to tube \#2 and immediately reconnect test tube \#1 and test tube \#2. (Mixture will look like its boiling or bubbling when diazald is reacting.)

15. Turn holder around so that test tubes are facing away from the analyst.

16. Lower hood to less than $1 / 4$ opening.

17. Reaction is complete when solution in test tube \#3 remains yellow for two minutes.

18. Turn off $\mathrm{N}_{2}$ and break rubber seal.

19. To destroy the un-reacted diazomethane in test tube \#2 by adding a small amount of water by pipette. The material in test tube \#2 will turn from yellow to clear.

20. Place the contents of test tube \#2 into a water soluble waste container.

21. Place a small amount of water in test tube \#1. The ether on the top layer can then be evaporated since the ether was checked for peroxide prior to use and the evaporation process will be performed over water.

\section{REFERENCES AND NOTES}

1. F. Arndt, in "Organic Synthesis: Collective Volume 2", A. H. Blatt, Ed., John Wiley \& Sons, Inc.: New York (1943) p 165.

2. Diazomethane Generation SOP (PDF); 2003.

3. H. M. Fales; T. M. Jaouni; J. F. Babashak, Analytical Chemistry, 45, 2302 (1973) 
4. J. March, "Advanced Organic Chemistry: Reactions, Mechanisms, and Structure", John Wiley \& Sons: New York, (1992) p 383.

5. F. Ngan; M. Toofan, Journal of Chromatographic Science, 29, 8 (1991)

6. D. Z. Brunengraber; B. J. McCabe; J. Katanik; S. F. Previs, Analytical Biochemistry, 306, 278 (2002)

7. G. A. Holmes; K. Rice; C. R. Snyder, Journal of Materials Science, 41, 4105 (2006)

Authors: Kathleen Flynn, Charles Guttman, and Gale A. Holmes 